\title{
The prevalence of global physical activity among young people: a systematic review for the Report Card Brazil 2018
}

\section{A prevalência de atividade física global entre jovens: uma revisão sistemática para o Report Card Brazil 2018}

Valter Cordeiro Barbosa Filho ${ }^{1}$

Rafael Martins da Costa ${ }^{2}$

Margarethe Thaisi Garro Knebel ${ }^{2}$

Bruno Nunes de Oliveira²

Camila Brasileiro de Araújo Silva ${ }^{3}$

Kelly Samara da Silva ${ }^{2}$
Abstract - The aim of this study was to summarize data on the prevalence of global physical activity (PA) among children and adolescents in Brazil. This systematic review included an electronic search in 8 databases (PubMed, Scopus, Web of Science, LILACS, SPORTDiscus, BIREME, Scielo, and Google Scholar) and a manual search in the references of retrieved studies. Observational studies that assessed global PA among Brazilian children and adolescents were included. A narrative approach toward results was adopted. An initial database search reached 3276 potentially relevant titles (2534 titles after duplicate analysis); 92 (62 different studies) met all inclusion criteria. Studies were carried out between 2001 and 2015; most studies were conducted in Southern and Northeastern Brazil. Three were nationwide surveys (4.8\%), but no studies included children aged 0-5 years. Eighteen different instruments for PA measurement were used; only two studies used objectively-measured PA. The most used definition was $\geq 300$ minutes/week of moderate-to-vigorous PA. The overall proportion of young people who were physically active ranged from $6.5 \%$ to $92.3 \%$. Fourteen studies (22.6\%) found a prevalence of $\geq 50 \%$. Three nationwide surveys reported the prevalence of physically active students ranging from $29.0 \%$ to $66.0 \%$. A wide variability of PA estimates were found. Important research gaps (e.g., studies with objectively-measured PA, children aged up to 6 years-old and in Northern Brazil) should be considered to develop new research studies in Brazil.

Key words: Adolescent; Adolescent health; Brazil; Exercise; Public health.

Resumo - Objetivou-se sumarizar dados sobre a prevalência de atividade física global (AF) entre crianças e adolescentes no Brasil. Esta revisão sistemática incluiu uma busca eletrônica em 8 bases de dados (PubMed, Scopus, Web of Science, LILACS, SPORTDiscus, BIREME, Scielo e Google Scholar) e uma busca manual nas referências de estudos recuperados. Estudos observacionais que avaliaram a AF global entre crianças e adolescentes brasileiros foram incluídos. Uma abordagem narrativa em relação aos resultados foi adotada. Uma pesquisa de banco de dados inicial atingiu 3276 títulos potencialmente relevantes (2534 títulos após análise duplicada); 92 (62 estudos diferentes) preencheram todos os critérios de inclusão. Os estudos foram realizados entre 2001 e 2015; a maioria dos estudos foi realizada no sul e nordeste do Brasil. Três foram pesquisas nacionais (4,8\%), mas nenhum estudo incluiu crianças de 0 a 5 anos. Dezoito instrumentos diferentes para medida de PA foram utilizados; apenas dois estudos usaram PA medida objetivamente. A definição mais utilizada foi $\geq 300$ minutos/semana de AF moderada a vigorosa. A proporçẫo geral de jovens que eram fisicamente ativos variou de 6,5\% a 92,3\%. Quatorze estudos $(22,6 \%)$ encontraram uma prevalência de $\geq 50 \%$. Três pesquisas nacionais relataram a prevalência de estudantes fisicamente ativos variando de 29,0\% a 66,0\%. Uma ampla variabilidade de estimativas de PA foi encontrada. Lacunas importantes de pesquisa (por exemplo, estudos com PA medida objetivamente, crianças de até 6 anos de idade e no norte do Brasil) devem ser consideradas para o desenvolvimento de novas pesquisas no Brasil.

Palavras-chave: Adolescente; Brasil; Exercício; Saúde do adolescente; Saúde pública.
1 Federal Institute of Ceará. Campus Boa Viagem. Boa Viagem, CE. Brazil.

2 Federal University of Santa Catarina. Florianópolis, SC. Brazil.

3 State University of Ceará. Fortaleza, CE. Brazil.

Received: April 25, 2018 Accepted: July 15, 2018 


\section{INTRODUCTION}

Physical activity is strongly associated with development, health, and quality of life among children and adolescents ${ }^{1}$. Despite this, only two out of ten adolescents meet physical activity guidelines (60 or more minutes per day of moderate-to-vigorous physical activity) worldwide ${ }^{2}$. Nationwide data show a similar trend in Brazilian adolescents ${ }^{3}$. Thus, policies and programs for physical activity promotion are urgent, and monitoring how and whether intervention strategies can improve physical activity practices among young populations is an important component of public health action.

In 2014, researchers, health professionals, and stakeholders built The Active Healthy Kids Global Alliance, a network focused on advancing physical activity among children and adolescents from around the world. For this, physical activity indicators (e.g., meeting physical activity guidelines, active transportation, and others) have been monitored and described in a document called a "Report Card", in order to be a useful tool for the advocacy of physical activity ${ }^{4}$ among young people. In 2016, data from 38 countries were included, including Brazil. A grade of $\mathrm{C}$ - (prevalence rates from $40 \%$ to $46 \%$ ) was given for global physical activity levels based on data from studies with Brazilian adolescents - this was one of the lowest grades for Brazil's 2016 Report Card5.

The present study aimed to systematically summarize the literature on the prevalence of global physical activity (based on international physical activity guidelines) among Brazilian children and adolescents. The summarized data were used to define the grade and recommendations for Brazil's 2018 Report Card for the global physical activity indicator.

\section{METHODOLOGICAL PROCEDURES}

\section{Measured Outcome}

In this systematic review, physical activity outcome was defined as any bodily movement produced by skeletal muscles that require energy expenditure ${ }^{6}$. Studies were considered whether they measured global physical activity (e.g., different domains combined (leisure, transportation, home and/or school combined), in order to follow the global physical activity guidelines ${ }^{7}$.

\section{Study Search Strategies}

A systematic search was conducted in electronic databases Medline (PubMed), Scopus, Web of Science (Web of Knowledge), LILACS (Literatura Latino-Americana em Ciências da Saúde), SPORTDiscus, BIREME (Biblioteca Regional de Medicina), Scielo, and Google Scholar in February 2018. The search strategy included four groups of descriptors: outcome (physical activity), physical activity evaluation methods, population (young people) and country (see Supplementary Material). The Boolean operator "OR" was used for intragroup combinations, and the "AND" operator was used for intergroup combinations. The truncation symbols (\$, * or “") 
specific to each database were also used to increase the range of searches for the descriptor variations. Searches were conducted with the descriptors in English and Portuguese, when required. The search of the electronic databases was supplemented by a screening of the reference list of retrieved articles in order to find potentially relevant titles and the personal library.

\section{Identification of Eligible Studies}

\section{- Selection Process}

The initial analysis was performed based on the reading of the manuscripts titles and, when there was doubt regarding the inclusion of the study, a reading of the abstract was carried out. After this analysis, articles were obtained in full text version and subsequently analyzed according to established selection criteria. Subsequently, the screening of the reference list was carried out. All the process was conducted by independent peers (RC/ $\mathrm{MK}$ or $\mathrm{BO} / \mathrm{CS}$ ), and a third author (KS or $\mathrm{VB}$ ) helped with disagreements.

\section{- Selection Criteria}

To be eligible for inclusion in this review, studies had to be: (I) articles published in peer-reviewed journals that were original research; (II) samples with Brazilian children and adolescents aged 0-18 years (or a mean age within these ranges, or separated data for individuals at this age range) (III) observational studies showing the prevalence of at least one of global physical activity (e.g., $\%$ of children and adolescents who accumulate a combined total of at least 60 minutes of daily moderate- to vigorous-intensity physical activity), regardless of whether the study dealt with this behavior as an exposure or an outcome; (IV) studies using different methods for PA assessment (e.g., self-report, structured interviews, objectively-measured PA, and steps per day); (V) a school- or population-based survey with information about the methodological procedures of representation of the target population (e.g., random sampling).

\section{- Data Extraction and Synthesis}

Information of each study was extracted by independent peers (RC/MK and $\mathrm{BO} / \mathrm{CS}$ ) and a third author (VB or $\mathrm{KS}$ ) helped with disagreements. Extracted information included: authors and year of publication, local of the study, year of data collect, age range, sample type, sample size, percentage of girls, instrument description, instruments characteristics and mode of administration, and physical activity cut-off definitions. A narrative approach for the results was adopted because the heterogeneity of the study's data (no meta-analyze was performed).

Also, the proportion of young people that were considered physically active (based on the physical activity definition in each study), as well as the proportion of this outcome for boys and girls separately, when presented.

Results of the studies were presented in alphabetical and chronological order by first author name and year of publication, respectively. The results were organized in order to present methodological aspects (Table 1) and the prevalence of global physical activity (Table 2) of the included studies. 


\section{RESULTS}

The initial database search identified a total of 3276 potential studies (2534 titles after duplicate analysis). Another 15 references were included based on the search in the reference lists of studies. After the title and abstract screening, 2284 references were excluded, and 265 references were taken forward to the full-text screening. In the full-text analysis, 147 references were excluded (exclusion reasons detailed in Figure 1), and 92 met the inclusion criteria (which included data from 62 different studies).
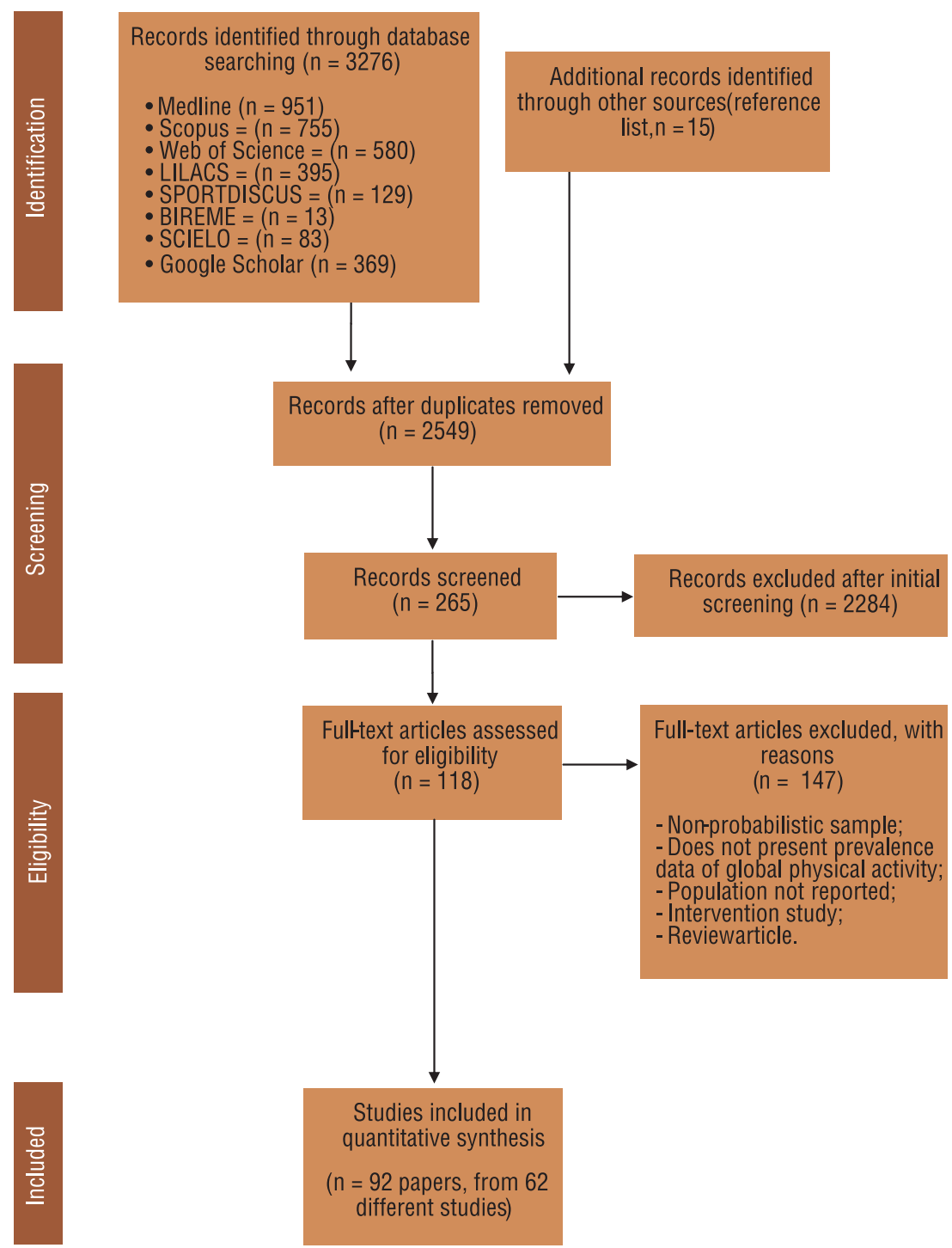

Figure 1: Search process results according PRISMA flow diagram

Included studies were carried out between $2001^{8-14}$ and 2015 them were performed in 2011 ( 12 studies $)^{16,17,26-31,18-25}$. Twenty studies were performed between 2011 and 2015 15,16,25-34,17,35-43,18-24. Most of included studies were located in the South and Northeast regions of Brazil: 22 $(35.5 \%)^{8,9,40-49,10,50-59,11,60-65,14,16,17,26,27,37}$ and $19(30.6 \%)^{9,12,29,31,38,39,66-71,13,72-}$ 
80,18-21,23,25,28 studies, respectively. Fifteen studies (24.2\%) were performed in the Southeast region, and three studies $(4.8 \%)^{22,24,81}$ were conducted in the Center-West region. No included reference was from the Northern region of Brazil. Three included studies were national surveys (4.8\%); one provided data from 17 capitals and the Federal District ${ }^{82}$ and the other two studies provided data from all of the 26 Brazilian capitals and the

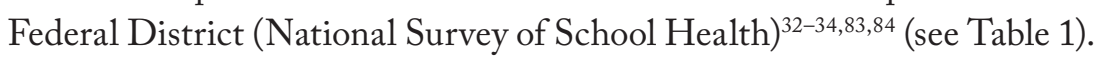

Almost all studies $(\mathrm{n}=59)$ were school-based surveys ${ }^{8,9}, 18-27,10,28,29$, $31-37,39,11,40-43,45-47,49-51,12,52-60,62,13,63-72,14,73-81,83,15,84-93,16,94-101,17$. Three ${ }^{61}$, 82,102 were population-based studies. In terms of age, three (4.9\%) studies included 6-10 year-old children $29,85,86,52$ (85.2\%) studies included 10-19

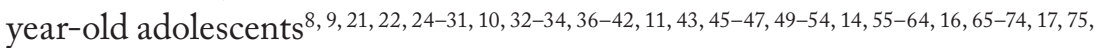
$76,78-84,87,18,88,90-94,96-99,19,101,102,20$, and six (9.8\%) studies included individuals from both age groups ${ }^{12,13,23,35,77,89,95}$.

Included studies presented 18 different instruments for physical activity measurement. Nineteen studies did not report the instruments used, and from these, only six studies reported information that the used instruments were validated ${ }^{10,19,47,63,71,76,78,86}$. The most used instruments were: the International Physical Activity Questionnaire (IPAQ 12 studies) $22,36,88,99,102,55,59,60,64,65,76,77,82$, the Global School-Health Survey instrument (GSHS, 8 studies) ${ }^{11,21,66-68,79,84,96,24,25,29,32,37,41,42,56}$, and the Physical Activity Questionnaire for Older Children and Adolescents (PAQ-C and PAQA, 10 studies) $)^{12,13,97,101,16-18,31,39,58,72,92}$. Finally, one study used pedometermeasured physical activity ${ }^{23}$ and one study used accelerometer-measured physical activity ${ }^{61}$ (Table 1).

The physical activity level was defined differently among the studies. The most used definition was based on the global recommendations for physical activity and health: the cut-off point of $\geq 300$ minutes/week of moderate-to-vigorous physical activity was used in 26 studies $^{8,} 10,45,47,55$, $57,59,60,63,64,69,70,11,71-73,76,79-81,83,84,86,22,88,89,91,93-95,97,98,26,27,29,36-38$, and 17 studies $^{20,21,39-43,49-53,24,54,56,58,61,65-68,74,75,25,85,96,28,31-35}$ used the cut-off point of $\geq 60$ minutes/day of moderate-to-vigorous physical activity (Table 1).

Table 1. Description of the studies on the global physical activity among Brazilian children and adolescents.

\begin{tabular}{|c|c|c|c|c|c|c|}
\hline Reference & Local & Sample type & $\begin{array}{c}\text { Sample } \\
\text { (\% of girls) }\end{array}$ & $\begin{array}{c}\text { Age } \\
\text { (years) }\end{array}$ & Instrument & $\begin{array}{l}\text { Mode of administra- } \\
\text { tion; recall time }\end{array}$ \\
\hline Abreu and Caiaffa ${ }^{82}$ & National & Population-based & $7169(54.2)$ & $15-24$ & IPAQ & Self-reported; PR-W \\
\hline Alves Júnior et al. ${ }^{a} 40$ & São José, SC & School-based & $820(56.0)$ & $14-17$ & $\begin{array}{c}\text { Undefined } \\
\text { Questionnaire }\end{array}$ & Uninformed; PR-W \\
\hline Andaki et al. ${ }^{85}$ & Viçosa, MG & School-based & $187(56.7)$ & 9.9 & $\begin{array}{c}\text { Undefined } \\
\text { Questionnaire }\end{array}$ & Self-reported; PR-W \\
\hline Andrade Neto et al. ${ }^{86}$ & $\begin{array}{c}\text { Santa Maria de } \\
\text { Jetibá, ES } \\
\text { Vitória, ES }\end{array}$ & School-based & $1770(55.1)$ & 7-10. & $\begin{array}{c}\text { Undefined } \\
\text { Questionnaire (V) }\end{array}$ & Not Described \\
\hline Arruda and Lopes ${ }^{87}$ & Lages, SC & School-based & $1024(0)$ & $10-17$ & $\begin{array}{l}\text { Bouchard diary } \\
\text { adapted }\end{array}$ & Self-reported \\
\hline Barros et al. ${ }^{66}$ & Recife, PE & School-based & $3764(59.5)$ & $14-19$ & GSHS & Self-reported; PR-W \\
\hline
\end{tabular}

Continue... 
.. continue

\begin{tabular}{|c|c|c|c|c|c|c|}
\hline Reference & Local & Sample type & $\begin{array}{l}\text { Sample } \\
\text { (\% of girls) }\end{array}$ & $\begin{array}{c}\text { Age } \\
\text { (years) }\end{array}$ & Instrument & $\begin{array}{l}\text { Mode of administra- } \\
\text { tion; recall time }\end{array}$ \\
\hline $\begin{array}{l}\text { Bastos, Araújo and } \\
\text { Hallal }^{63}\end{array}$ & Pelotas, RS & School-based & $857(52)$ & $10-19$ & $\begin{array}{c}\text { Undefined } \\
\text { Questionnaire (V) }\end{array}$ & Not Described \\
\hline Beck et al. ${ }^{45}$ & Três de Maio, RS & School-based & $660(52)$ & $14-19$ & $\begin{array}{c}\text { Undefined } \\
\text { Questionnaire }\end{array}$ & Not Described \\
\hline Bergmann et al. ${ }^{\mathrm{b} 16}$ & Uruguaiana, RS & School-based & $1455(50.9)$ & $10-17$ & $P A Q-C$ and $P A Q-A$ & Self-reported; PR-W \\
\hline Campos et al. ${ }^{46}$ & Curitiba, PR & School-based & $497(47.6)$ & $14-19$ & $\begin{array}{l}\text { Undefined } \\
\text { Questionnaire }\end{array}$ & Self-reported; HA-W \\
\hline Carvalho et al. ${ }^{.7}$ & Nordeste & School-based & $421(59.6)$ & $9-19$ & $I P A Q$ & Self-reported; PR-W \\
\hline Cavalcanti et al. ${ }^{67}$ & Recife, PE & School-based & 4138 (59.8) & $14-19$ & GSHS & Not Described \\
\hline $\begin{array}{l}\text { Ceschini, Andrade } \\
\text { and Figueira Júnior }{ }^{36}\end{array}$ & São Paulo, SP & School-based & $1844(46.5)$ & $15-20$ & IPAQ & Self-reported; PR-W \\
\hline Ceschini et al. ${ }^{88}$ & São Paulo, SP & School-based & $3845(52.6)$ & $14-19$ & IPAQ & Self-reported; PR-W \\
\hline Coelho et al. ${ }^{89}$ & OuroPreto, MG & School-based & $661(47.8)$ & $10-14$ & $\begin{array}{l}\text { Undefined } \\
\text { Questionnaire }\end{array}$ & Not Described \\
\hline Cureau et al. ${ }^{26}$ & Santa Maria, RS & School-based & $1132(53.9)$ & $14-19$ & $\begin{array}{l}\text { Undefined } \\
\text { Questionnaire }\end{array}$ & Not Described \\
\hline $\begin{array}{l}\text { Dambros, Lopes and } \\
\text { Santos }^{47}\end{array}$ & Santa Maria, RS & School-based & $450(45.3)$ & $14-18$ & $\begin{array}{c}\text { Undefined } \\
\text { Questionnaire (V) }\end{array}$ & Not Described \\
\hline Dumith et al. ${ }^{20}$ & Caracol, PI & School-based & $1112(53.3)$ & $13-19$ & $\begin{array}{l}\text { Undefined } \\
\text { Questionnaire }\end{array}$ & Self-reported; HA-W \\
\hline Farias Júnior ${ }^{14}$ & Florianópolis, SC & School-based & 1949 (50.6) & $12-18$ & $\begin{array}{l}\text { Bouchard diary } \\
\text { adapted }\end{array}$ & $\begin{array}{c}\text { Self-reported; } 3 \\
\text { days }\end{array}$ \\
\hline FariasJúnior ${ }^{78}$ & João Pessoa, PB & School-based & $2566(55.9)$ & $14-18$ & $\begin{array}{c}\text { Undefined } \\
\text { Questionnaire (V) }\end{array}$ & Self-reported; PR-W \\
\hline Farias Júnior et al. ${ }^{11}$ & Santa Catarina & School-based & $5028(59.3)$ & $15-19$ & GSHS & Self-reported; HA-W \\
\hline Farias Júnior et al. ${ }^{79}$ & João Pessoa, PB & School-based & $2874(57.8)$ & $14-19$ & GSHS & Self-reported; PR-W \\
\hline Farias Júnior et al. ${ }^{969}$ & João Pessoa, PB & School-based & $2874(57.8)$ & $14-19$ & $\begin{array}{l}\text { Undefined } \\
\text { Questionnaire }\end{array}$ & Not Described \\
\hline FariasJúnior et al. ${ }^{973}$ & João Pessoa, PB & School-based & $2859(57.8)$ & $14-19$ & $\begin{array}{l}\text { PACE and } \\
\text { QAFA }\end{array}$ & Self-reported; PR-W \\
\hline $\begin{array}{l}\text { Farias Júnior, Reis } \\
\text { and Hallal }{ }^{9} 70\end{array}$ & João Pessoa, PB & School-based & $2859(57.8)$ & 16,5 & $\begin{array}{l}\text { Undefined } \\
\text { Questionnaire }\end{array}$ & Self-reported; PR-D \\
\hline Fermino et al. ${ }^{\mathrm{h} 49}$ & Curitiba, PR & School-based & $1518(59.2)$ & $14-18$ & $\begin{array}{l}\text { Undefined } \\
\text { Questionnaire }\end{array}$ & Self-reported; HA-W \\
\hline Freire et al. ${ }^{90}$ & Montes Claros, MG & School-based & $763(48.2)$ & $15-19$ & $\begin{array}{c}\text { Undefined } \\
\text { Questionnaire }\end{array}$ & Self-reported \\
\hline Guilherme et al. ${ }^{37}$ & Paranavaí, PR & School-based & $566(49.3)$ & $10-14$ & GSHS & Not Described \\
\hline Hallal et al. ${ }^{d 84}$ & National & School-based & 60.973 & $13-15$ & GSHS & Self-reported; PR-W \\
\hline Leão et al. ${ }^{f 19}$ & Aracajú, SE & School-based & $2030(63.2)$ & $13-18$ & $\begin{array}{c}\text { Undefined } \\
\text { Questionnaire (V) }\end{array}$ & Not Described \\
\hline Legnani et al. ${ }^{56}$ & Foz do Iguacu, PR & School-based & $453(59)$ & $15-18$ & GSHS & Not Described \\
\hline $\begin{array}{l}\text { Leites, Bastos and } \\
\text { Bastos }^{57}\end{array}$ & Porto Alegre, RS & School-based & 967 (49.6) & $10-19$ & $\begin{array}{l}\text { Undefined } \\
\text { Questionnaire }\end{array}$ & Not Described \\
\hline Lima et al. ${ }^{\mathrm{h} 64}$ & Curitiba, PR & School-based & $1474(59)$ & $14-18$ & $\begin{array}{l}\text { Undefined } \\
\text { Questionnaire }\end{array}$ & Not Described \\
\hline Lima and Silva ${ }^{a} 41$ & São José, SC & School-based & $1103(54.5)$ & $14-19$ & GSHS & Self-reported; PR-W \\
\hline Loche $^{10}$ & Santa Catarina & School-based & $5083(59.4)$ & $15-19$ & $\begin{array}{c}\text { Undefined } \\
\text { Questionnaire (V) }\end{array}$ & Not Described \\
\hline Lucena et al. ${ }^{971}$ & João Pessoa, PB & School-based & $2874(57.8)$ & $14-19$ & $\begin{array}{c}\text { Undefined } \\
\text { Questionnaire (V) }\end{array}$ & Self-reported; PR-W \\
\hline Malta et al. ${ }^{d 83}$ & National & School-based & $60.973(53.3)$ & $13-14$ & $\begin{array}{l}\text { Undefined } \\
\text { Questionnaire }\end{array}$ & Not Described \\
\hline Malta et al. ${ }^{1} 32$ & National & School-based & $109.104(52.1)$ & $13-16$ & GSHS & Not Described \\
\hline
\end{tabular}




\begin{tabular}{|c|c|c|c|c|c|c|}
\hline Reference & Local & Sample type & $\begin{array}{l}\text { Sample } \\
\text { (\% of girls) }\end{array}$ & $\begin{array}{c}\text { Age } \\
\text { (years) }\end{array}$ & Instrument & $\begin{array}{l}\text { Mode of administra- } \\
\text { tion; recall time }\end{array}$ \\
\hline $\begin{array}{l}\text { Martins, Lima and } \\
\text { Silva }^{\mathrm{a} 42}\end{array}$ & São José, SC & School-based & $1132(54.2)$ & $14-19$ & GSHS & Self-reported; HA-W \\
\hline Matsudo et al. ${ }^{102}$ & São Paulo & Population-based & $2001(52.4)$ & $14-77$ & IPAQ & Self-reported; PR-W \\
\hline Meireles et al. ${ }^{91}$ & Belo Horizonte, MG & School-based & $1042(47.2)$ & $11-17$ & $\begin{array}{c}\text { Undefined } \\
\text { Questionnaire }\end{array}$ & Uninformed \\
\hline $\begin{array}{l}\text { Mendonça and Farias } \\
\text { Júnior }{ }^{72}\end{array}$ & João Pessoa, PB & School-based & $2859(57.8)$ & $14-19$ & $P A Q-A$ & Self-reported; PR-D \\
\hline Mendonça et al. ${ }^{9} 80$ & João Pessoa, PB & School-based & $2874(57.8)$ & $14-19$ & $\begin{array}{c}\text { Undefined } \\
\text { Questionnaire }\end{array}$ & Self-reported; PR-D \\
\hline $\begin{array}{l}\text { Menezes and } \\
\text { Duarte }^{21}\end{array}$ & Sergipe & School-based & 3992 (63.1) & $14-19$ & GSHS & Not Described \\
\hline Moraes et al. ${ }^{59}$ & Maringá, PR & School-based & $991(54.5)$ & $14-18$ & IPAQ & Self-reported; PR-W \\
\hline Moraes and Falcão ${ }^{58}$ & Maringá, PR & School-based & $991(54.5)$ & $14-18$ & $P A Q-A$ & Not Described \\
\hline Nakamura et al..$^{92}$ & Rio Claro, SP & School-based & $467(54)$ & $14-17$ & $P A Q-C$ & Self-reported; PR-W \\
\hline Nascente et al. ${ }^{22}$ & Goiânia, G0 & School-based & $862(52.8)$ & $14-18$ & IPAQ & Self-reported; PR-W \\
\hline $\begin{array}{l}\text { Nascimento-Ferreira } \\
\text { et al. } .^{103}\end{array}$ & Imperatriz, MA & School-based & $869(53.4)$ & $14-19$ & $P A Q-A$ & Self-reported; PR-D \\
\hline Nunes et al. ${ }^{\mathrm{a} 43}$ & São José, SC & School-based & $916(55.4)$ & $14-19$ & YRBSS & Self-reported; PR-W \\
\hline $\begin{array}{l}\text { Pelegrini and Pet- } \\
\text { roskik60 }\end{array}$ & Florianópolis, SC & School-based & $595(67.1)$ & $14-18$ & IPAQ & Self-reported; PR-W \\
\hline $\begin{array}{l}\text { Pereira, Bergmann } \\
\text { and Bergmannn } 17\end{array}$ & Uruguaiana, RS & School-based & 1455 (50.9) & $10-17$ & $P A Q-C$ and $P A Q-A$ & $\begin{array}{c}\text { Self-reported; PR-D } \\
\text { e PR-W }\end{array}$ \\
\hline Peres et al. ${ }^{93}$ & Piracicaba, SP & School-based & $269(56.1)$ & $10-14$ & QAFA & Self-reported; HA-M \\
\hline Petribú et al. ${ }^{\mathrm{m} 74}$ & Caruaru, PE & School-based & $600(62.5)$ & $15-20$ & COMCAP & Not Described \\
\hline Prado et al. ${ }^{\mathrm{h} 53}$ & Curitiba, PR & School-based & $1469(59.2)$ & $14-18$ & YRBSS & Self-reported; PR-W \\
\hline Quadros et al. ${ }^{23}$ & Amargosa, BA & . School-based & $1139(55.6)$ & $6-18$ & Pedometer & $\begin{array}{l}\text { Monitoramento; } \\
\text { PR-W }\end{array}$ \\
\hline Raizel et al. ${ }^{24}$ & Cuiabá, MT & School-based & $364(59)$ & $12-19$ & GSHS & Not Described \\
\hline Regis et al. ${ }^{\mathrm{n}}{ }^{25}$ & Pernambuco & School-based & $6234(59.7)$ & $14-19$ & GSHS & Not Described \\
\hline Reichert et al. ${ }^{61}$ & Pelotas, RS & Population-based & $457(47.9)$ & $12-14$ & Accelerometer & $\begin{array}{c}\text { Monitoramento; } \\
\text { PR-W }\end{array}$ \\
\hline Reis et al. ${ }^{\mathrm{h}} 54$ & Curitiba, PR & School-based & 1650 (59.6) & $14-18$ & $\begin{array}{c}\text { Undefined } \\
\text { Questionnaire }\end{array}$ & Self-reported; PR-W \\
\hline Rezende et al. ${ }^{i 33}$ & National & School-based & $109.104(57)$ & $14-15$ & $\begin{array}{c}\text { Undefined } \\
\text { Questionnaire }\end{array}$ & Self-reported; HA-W \\
\hline Rivera et al. ${ }^{012}$ & Maceió, AL & School-based & $1253(56.3)$ & $7-17$ & $P A Q-C$ & Self-reported; PR-W \\
\hline Rodrigues et al. ${ }^{81}$ & Cuiabá, MT & School-based & $1139(55.6)$ & $14-19$ & $\begin{array}{c}\text { Undefined } \\
\text { Questionnaire }\end{array}$ & Not Described \\
\hline Romanzini et al. ${ }^{55}$ & Londrina, PR & School-based & $644(61.6)$ & $16,4,0$ & IPAQ & Self-reported; PR-W \\
\hline Romero et al. ${ }^{194}$ & Piracicaba, SP & School-based & $328(54.3)$ & $10-15$ & QAFA & $\begin{array}{c}\text { Self-reported; PR-W } \\
\text { e PR-Y }\end{array}$ \\
\hline $\begin{array}{l}\text { Salvador, Kitoko and } \\
\text { Gambardella }{ }^{95}\end{array}$ & Vitória, ES & School-based & $400(47.3)$ & $8-17$ & $\begin{array}{c}\text { Undefined } \\
\text { Questionnaire }\end{array}$ & Self-reported; PR-Y \\
\hline Santos et al. ${ }^{\mathrm{h} 51}$ & Curitiba, PR & School-based & 1615 (59.69) & $14-18$ & $\begin{array}{c}\text { Undefined } \\
\text { Questionnaire }\end{array}$ & Not Described \\
\hline Santos et al. ${ }^{35}$ & Uberaba, MG & School-based & $649(52.1)$ & $9-12$ & LAF & Self-reported; PR-D \\
\hline Santos et al. ${ }^{n} 96$ & Pernambuco & School-based & $6264(59.7)$ & $14-19$ & GSHS & Not Described \\
\hline Santos et al. ${ }^{31}$ & Pernambuco & School-based & 4207 (59.8) & $14-19$ & $P A Q-C^{*}$ & Self-reported; PR-W \\
\hline Silva $(2015)^{i} 34$ & National & School-based & $\begin{array}{c}109.104 \\
(52.2)\end{array}$ & $13-15$ & $\begin{array}{c}\text { Undefined } \\
\text { Questionnaire }\end{array}$ & Self-reported; PR-W \\
\hline Silva and Silva ${ }^{p 28}$ & Aracajú, SE & School-based & 2057 (62.7) & $13-18$ & YRBSS & Self-reported; PR-W \\
\hline Silva et al. ${ }^{013}$ & Maceió, AL & School-based & $706(56.3)$ & $7-17$ & $P A Q$ & Self-reported; PR-W \\
\hline
\end{tabular}

Continue. 
.. continue

\begin{tabular}{|c|c|c|c|c|c|c|}
\hline Reference & Local & Sample type & $\begin{array}{c}\text { Sample } \\
\text { (\% of girls) }\end{array}$ & $\begin{array}{c}\text { Age } \\
\text { (years) }\end{array}$ & Instrument & $\begin{array}{l}\text { Mode of administra- } \\
\text { tion; recall time }\end{array}$ \\
\hline Silva et al. ${ }^{e 8}$ & Santa Catarina & School-based & $5028(59.3)$ & $15-19$ & COMPAC & Self-reported; HA-W \\
\hline Silva et al. ${ }^{97}$ & Aracajú, SE & School-based & 1028 (56.7). & $13-16$ & $P A Q-C$ & Self-reported; PR-W \\
\hline Silva et al. ${ }^{\mathrm{e}}$ & Santa Catarina & School-based & $5028(59.3)$ & $15-19$ & COMPAC & Self-reported; HA-W \\
\hline Silva et al. ${ }^{p} 18$ & Aracajú, SE & School-based & $2105(63.0)$ & $13-18$ & YRBSS & Self-reported; PR-W \\
\hline Silva et al. ${ }^{27}$ & Santa Catarina & School-based & $6529(53.9)$ & $15-19$ & COMPAC & Self-reported; HA-W \\
\hline Silva et al. ${ }^{64}$ & Ponta Grossa, PR & School-based & $653(57,9)$ & $14-19$ & IPAQ & Self-reported; PR-W \\
\hline Silva et al. ${ }^{k 65}$ & Florianópolis, SC & School-based & $696(62.1)$ & $15-18$ & IPAQ & Self-reported; PR-W \\
\hline Silva et al..$^{15}$ & Arapiraca, SE & School-based & $571(52.7)$ & $8-10$ & QUAFDA & Self-reported; PR-D \\
\hline $\begin{array}{l}\text { Silva, Silva and } \\
\text { Oliveira }^{\dagger 18}\end{array}$ & Aracajú, SE & School-based & $2259(62.3)$ & $13-18$ & $P A Q-C$ & Self-reported; PR-W \\
\hline $\begin{array}{l}\text { Silva, Smith-Menez- } \\
\text { es and Duarte }\end{array}$ & Sergipe & School-based & 3992 (61.3) & $14-19$ & GSHS & Not Described \\
\hline Souza $^{98}$ & Canoas, RS & School-based & $293(66.9)$ & 17 & IPAQ & Self-reported; PR-W \\
\hline Souza et al. ${ }^{76}$ & Salvador, BA & School-based & $694(52.7)$ & $10-14$ & $\begin{array}{c}\text { Undefined } \\
\text { Questionnaire (V) }\end{array}$ & Not Described \\
\hline Souza et al. ${ }^{\text {h } 52}$ & Curitiba, PR & School-based & $1698(59.4)$ & $14-18$ & $\begin{array}{c}\text { Undefined } \\
\text { Questionnaire }\end{array}$ & Self-reported; HA-W \\
\hline Straatmann e Veiga ${ }^{99}$ & Niterói, RJ & School-based & $.697(59.6)$ & $12-19$ & IPAQ & Self-reported; PR-W \\
\hline Suñe et al. ${ }^{62}$ & $\begin{array}{c}\text { Capão da Canoa, } \\
\text { RS }\end{array}$ & School-based & $719(50.2)$ & $11-13$ & $\begin{array}{c}\text { Questionnaire on } \\
\text { habitual physical } \\
\text { activities (Russell } \\
\text { R. Pate) }\end{array}$ & Not Described \\
\hline Tassitano et al. ${ }^{\mathrm{m} 75}$ & Caruaru, PE & School-based & $600(62.5)$ & $15-20$ & COMCAP & Not Described \\
\hline Tenório et al. ${ }^{\mathrm{c} 68}$ & Pernambuco & School-based & $4210(59.8)$ & $14-19$ & GSHS & Not Described \\
\hline Vieira et al. ${ }^{100}$ & Natal, RN & School-based & 347 (72) & $12-18$ & $\begin{array}{c}\text { Undefined } \\
\text { Questionnaire }\end{array}$ & Not Described \\
\hline Vitta et al. ${ }^{101}$ & Bauru, SP & School-based & $524(46.9)$ & $10-14$ & $P A Q-C$ & Self-reported; PR-W \\
\hline
\end{tabular}

Note. Superscript equal letters over author's name refer to distinct articles from the same study; Undefined Questionnaire: Original instrument not identified by the authors, but questions were reported; (V): Questionnaire presented criterion, content and/or construct validity; States of Brazil: AL: Alagoas; BA: Bahia; DF: Distrito Federal; ES: Espírito Santo; GO: Goiás; MA: Maranhão; MG: Minas Gerais; MT: Mato Grosso; PB: Paraíba; PE: Pernambuco; PI: Piauí; PR: Paraná; RJ: Rio de Janeiro; RN: Rio Grande do Norte; RS: Rio Grande do Sul; SC: Santa Catarina; SE: Sergipe; SP: São Paulo. (PAQ-C): Physical Activity Questionnaire for Older Children; (PAQ-A): Physical Activity Questionnaire for Adolescents; (PAQ-C*): Activity Questionnaire for Children and Adolescents; (GSHS): Global School-based Student Health Survey; (YRBSS): Youth Risk Behavior Surveillance; (Saúde na Boa): Good Health Questionnaire; (QAFA): Physical Activity Questionnaire for Adolescents; (LAF): List of Physical Activities; (PAQ): Physical Activity Questionaire; (COMCAP): Risk Behaviors in Catarinese Adolescents; (QUAFDA): Previous Day Physical Activity Questionnaire; (IPAQ): International Physical Activity Questionnaire; (COMPAC): Behaviors of Catarinense Adolescents; (PeNSE 2012): National School Health Survey. a - Brazilian Guide for Evaluation of Health-Related Physical Fitness and Life Habits - Stage I; b - Estudo em Uruguaiana/RS - protocol do Comitê de Ética em Pesquisa: 042/2010; c - Estilos de Vida e Comportamentos de Risco à Saúde em Estudantes do Ensino Médio no Estado de Pernambuco; d - Pesquisa Nacional de Saúde do Escolar, edição 2009 - PeNSE 2009; e - Estilo de Vida e Comportamentos de Risco dos Jovens Catarinenses, edição 2001 - COMPAC 2001; f - Estudo em Sergipe - protocolo do Comitê de Ética em Pesquisa da Universidade Federal de Sergipe (CEP/UFS): 5724.0.000.107-10; $\mathrm{g}$ - Physical activity level and associated factors among high school adolescents in João Pessoa, city, - PB: an ecological approach.; h - Determinantes da Atividade Física e Obesidade em Escolares do Ensino Médio da Rede Pública da Cidade de Curitiba, Paraná, Brasil.; i - Pesquisa Nacional de Saúde do Escolar, edição 2012 - PeNSE 2012; j - Estudo em Maringá/PR - Aprovado pelo Comitê de Ética em Pesquisa com Seres Humanos do Centro Universitário de Maringá.; k - Níveis de atividade física, aptidão física e comportamento social relacionados à saúde em escolares de Florianópolis, SC.; I - Consumo dietético e AF como determinantes das mudanças do IMC de uma coorte de adolescentes matriculados da rede pública de ensino da cidade de Piracicaba/SP.; $m$ - Estudo em Caruaru/PE - protocolo do Comitê de Ética em Pesquisa com Seres Humanos da Faculdades ASCES: 44/2007.; $n$ - Physical activity practice and health risk behaviors in high school students in the State of Pernambuco: a temporal trend study.; 0 - Estudo em Maceió-AL - Aprovado pelo Comitê de Ética do Hospital Universitário da Universidade Federal de Alagoas.; p Estudo em Aracajú/SE - Aprovado pelo Ethics Committee on Human Research of the Federal University of Sergipe.

The overall proportion of young people who were physically active ranged from $6.5 \%{ }^{12}$ to $92.3 \%{ }^{64}$. Considering the estimate of 41 studies ${ }^{8,9}$, $25,27-29,31-34,36,37,10,45-47,49-55,11,56-61,63,65-67,14,68-76,78,18,79,80,83-86,88,92-94,19,96,97$, $99,101-103,20,21,24$ that provided gender-stratified data, the prevalence of being 
physically active ranged from $0.3 \%{ }^{9}$ to $84.6 \%{ }^{86}$ in girls, and from $2.0 \%{ }^{9}$ to $93.1 \%{ }^{93}$ in boys. Fourteen studies $(22.6 \%)$ demonstrated a proportion of active individuals $\geq 50 \% 8,9,65,69-75,77,79,10,80,82,87,89,95,11,35,38,55,60,61,64$. Among the three nationwide surveys included in this review, the proportions of physically active young people were $66.0 \%$ (Cancer National Institute 2002/2003) ${ }^{82}$, 43.1\% (National Survey of School Health-2009) ${ }^{84}$ and 29.0\% (National Survey of School Health-2012) ${ }^{33}$ (Table 2).

Considering the results of adolescents according to age groups, the proportion of physically active children (up to 6-10 year-olds exclusively) ranged from $10.9 \%^{15}$ to $29.2 \%{ }^{89}$. Studies with adolescents exclusively (10 years or older) presented proportions that ranged from $8.3 \%{ }^{17}$ to $92.3 \%{ }^{64}$ (Table 2).

Included studies that used the cutoff point of $\geq 300$ minutes/week of moderate-to vigorous physical activity presented proportions of physically active individuals that ranged from $18.4 \%{ }^{86}$ to $92.3 \%{ }^{64}$. Studies using the cutoff point of $\geq 60$ minutes/day moderate-to vigorous physical activity presented proportions of physically active individuals that ranged from $12.6 \%{ }^{20}$ to $68.2 \%{ }^{65}$. Studies using the cut-off score for PAQ-C/PAQ-A scores presented proportions ranging from $6.5 \%{ }^{12}$ to $23.5 \%{ }^{101}$. Studies that used the cutoff point of the IPAQ short presented proportions varying from $66.0 \%{ }^{82}$ to $69.8 \%{ }^{77}$.

Regarding objective measurements of PA, the population-based study that used accelerometers ${ }^{61}$ was conducted in South Brazil with 457 adolescents who were 12-14 years old. This study found overall prevalence of physically active of $61.3 \%{ }^{61}$. A school-based study with 1139 6-8-year-old students from Northeastern Brazil the used pedometer-measured physical activity found an overall prevalence of $35.7 \%$ physically active individuals ${ }^{23}$ (Table 2).

Trends in the prevalence of physical activity were observed in three studies, which presented two waves of data collection. The National School Health Survey (2009 and 2012) described percentages of physically active students of $43.1 \%$ in $2009^{83,84}$ to $29.0 \%$ in $2012^{33}$. However, the number of questions used to define physical activity level was different between these surveys. The Health Risk Behaviors Project (COMPAC study, a study in Santa Catarina state) showed a prevalence of physically active boys and girls of $72.1 \%$ and $71.2 \%$ in $2001^{9}$, and $39.3 \%$ and $23.7 \%$ in 2011 , respectively ${ }^{27}$. A statewide survey in Pernambuco state presented the prevalence of physically active students of $34.9 \%$ in $2006^{31,68}$ and $35.1 \%$ in $2011^{96}$.

Table 2. Description of prevalence of physical activity (\%) in studies including Brazilian children and adolescents.

\begin{tabular}{|c|c|c|c|c|c|}
\hline \multirow{2}{*}{ Reference } & \multirow{2}{*}{ Local } & \multirow{2}{*}{ Definition } & \multicolumn{3}{|c|}{ Prevalence of Physical Activity } \\
\hline & & & All & Boys & Girls \\
\hline Abreu and Caiaffa ${ }^{82}$ & National & IPAQ version 8 definition. & $66.0 \%$ & n.a. & n.a. \\
\hline $\begin{array}{l}\text { Alves Júnior et } \\
\text { al. }^{a 40}\end{array}$ & São José, SC & $\geq 60 \mathrm{~min} . /$ day of MVPA & $22.9 \%$ & n.a. & n.a. \\
\hline Andaki et al. ${ }^{85}$ & Viçosa, MG & $\geq 60 \mathrm{~min} . /$ day of MVPA & n.a. & $68.1 \%$ & $36.7 \%$ \\
\hline $\begin{array}{l}\text { Andrade Neto et } \\
\text { al. }{ }^{86}\end{array}$ & $\begin{array}{c}\text { Santa Maria } \\
\text { de Jetibá, ES } \\
\text { Vitória, ES }\end{array}$ & $\geq 300$ min./weekof MVPA & $18.4 \%$ & $\begin{array}{l}\text { Urban area: } 75.5 \% \\
\text { Rural area: } 90.8 \%\end{array}$ & $\begin{array}{l}\text { Urban area: } 69.2 \% \\
\text { Rural area: } 84.6 \%\end{array}$ \\
\hline
\end{tabular}

Continue... 
.. continue

\begin{tabular}{|c|c|c|c|c|c|}
\hline \multirow{2}{*}{ Reference } & \multirow{2}{*}{ Local } & \multirow{2}{*}{ Definition } & \multicolumn{3}{|c|}{ Prevalence of Physical Activity } \\
\hline & & & All & Boys & Girls \\
\hline $\begin{array}{l}\text { Arruda and Lopes } \\
87\end{array}$ & Lages, SC & $>39,9 \mathrm{kcal} / \mathrm{kg} /$ day & $58.8 \%$ & n.a. & n.a. \\
\hline Barros et al. $\# 66$ & Recife, PE & $\geq 60 \mathrm{~min} . /$ day of MVPA & $34.0 \%$ & $40.1 \%$ & $29.8 \%$ \\
\hline $\begin{array}{l}\text { Bastos, Araújo and } \\
\text { Hallal }^{\# 63}\end{array}$ & Pelotas, RS & $\geq 300$ min./week of MVPA & $30.2 \%$ & $43.5 \%$ & $17.9 \%$ \\
\hline Beck et al. $\# 45$ & Três de Maio, RS & $\geq 300$ min./weekof MVPA & $38.8 \%$ & $47.6 \%$ & $30.6 \%$ \\
\hline Bergmann et al. ${ }^{\mathrm{b}} 16$ & Uruguaiana, RS & $\begin{array}{c}P A Q-C \text { / PAQ-A: (4 and } 5 \\
\text { points) }\end{array}$ & $08.3 \%$ & n.a. & n.a. \\
\hline Campos et al. ${ }^{*} 46$ & Curitiba, PR & $\geq 37 \mathrm{kcal} / \mathrm{kg} / \mathrm{dia}$ & n.a. & $82.7 \%$ & $77.4 \%$ \\
\hline Carvalho et al. $\# 77$ & Northeast & $\begin{array}{l}\text { IPAQ short version; } \\
\text { definition. }\end{array}$ & $69.8 \%$ & n.a. & n.a. \\
\hline Cavalcanti et al. ${ }^{c 67}$ & Recife, PE & $\geq 60 \mathrm{~min} . /$ day of MVPA & $5.3 \%$ & $4.1 \%$ & $6.5 \%$ \\
\hline $\begin{array}{l}\text { Ceschini, Andrade } \\
\text { and Figueira } \\
\text { Júnior }{ }^{36}\end{array}$ & São Paulo, SP & $\geq 300$ min./week of MVPA & $36.1 \%$ & $39.4 \%$ & $32.2 \%$ \\
\hline Ceschini et al. $\# 88$ & São Paulo, SP & $\geq 300$ min./week of MVPA & $37.5 \%$ & $50.3 \%$ & $25.9 \%$ \\
\hline Coelho et al. ${ }^{89}$ & Ouro Preto, MG & $\geq 300$ min./week of MVPA & $\begin{array}{l}29.2 \% \text { (6-9 years } \\
\text { old). } \\
70.8 \% \text { (10-14 years } \\
\text { old) }\end{array}$ & n.a. & n.a. \\
\hline Cureau et al. ${ }^{26}$ & Santa Maria, RS & $\geq 300$ min./week of MVPA & $46.5 \%$ & n.a. & n.a. \\
\hline $\begin{array}{l}\text { Dambros, Lopes } \\
\text { and Santos }{ }^{* 7}\end{array}$ & Santa Maria, RS & $\geq 300$ min./week of MVPA & n.a. & $75.0 \%$ & $60.0 \%$ \\
\hline Dumith et al. ${ }^{20}$ & Caracol, PI & $\geq 60 \mathrm{~min} . /$ day of MVPA & $12.6 \%$ & $20.2 \%$ & $05.9 \%$ \\
\hline Farias Júnior\# 14 & Florianópolis, SC & $\geq 37 \mathrm{kcal} / \mathrm{kg} / \mathrm{day}$ & $37.4 \%$ & $48.6 \%$ & $26.5 \%$ \\
\hline Farias Júnior $\# 78$ & João Pessoa, PB & $\geq 37 \mathrm{kcal} / \mathrm{kg} / \mathrm{day}$ & n.a. & $54.5 \%$ & $35.8 \%$ \\
\hline $\begin{array}{l}\text { Farias Júnior et } \\
\text { al. }{ }^{\text {e\# } 11}\end{array}$ & Santa Catarina & $\geq 300$ min./week of MVPA & $63.5 \%$ & $73.9 \%$ & $56.3 \%$ \\
\hline $\begin{array}{l}\text { Farias Júnior et } \\
\text { al. }{ }^{79}\end{array}$ & João Pessoa, PB & $\geq 300$ min./week of MVPA & $50.2 \%$ & $66.3 \%$ & $38.5 \%$ \\
\hline $\begin{array}{l}\text { Farias Júnior et } \\
\text { al. }{ }^{69}\end{array}$ & João Pessoa, PB & $\geq 300$ min./week of MVPA & $50.2 \%$ & $66.3 \%$ & $38.5 \%$ \\
\hline $\begin{array}{l}\text { Farias Júnior et } \\
\text { al. }{ }^{73}\end{array}$ & João Pessoa, PB & $\geq 300$ min./week of MVPA & $\begin{array}{c}20.1 \% \text { (simplified) } \\
50.2 \% \text { (detailed) }\end{array}$ & $\begin{array}{c}28.3 \% \text { (simplified); } \\
66.3 \% \text { (detailed) }\end{array}$ & $\begin{array}{c}14.1 \% \text { (simplified); } \\
38.5 \% \text { (detailed) }\end{array}$ \\
\hline $\begin{array}{l}\text { Farias Júnior, Reis } \\
\text { and Hallal }{ }^{70}\end{array}$ & João Pessoa, PB & $\geq 300$ min./week of MVPA & n.a. & $66.3 \%$ & $38.5 \%$ \\
\hline Fermino et al. ${ }^{\mathrm{h}}{ }^{49}$ & Curitiba, PR & $\geq 60 \mathrm{~min} . /$ day of MVPA & $14.5 \%$ & $22.3 \%$ & $09.1 \%$ \\
\hline Freire et al..$^{90}$ & $\begin{array}{c}\text { Montes Claros, } \\
\text { MG }\end{array}$ & $\begin{array}{l}\text { Frequency of physi- } \\
\text { cal activity practice } \\
\text { ("always"/“frequently"). }\end{array}$ & $45.2 \%$ & n.a. & n.a. \\
\hline Guilherme et al. ${ }^{\# 37}$ & Paranavaí, PR & $\geq 300$ min./week of MVPA & n.a. & $46.9 \%$ & $53.1 \%$ \\
\hline Hallal et al. ${ }^{\mathrm{d}} 84$ & National & $\geq 300$ min./week of MVPA & $43.1 \%$ & $56.2 \%$ & $31.3 \%$ \\
\hline Leão et al. ${ }^{f 19}$ & Aracajú, SE & $\begin{array}{c}\mathrm{PAQ}-\mathrm{C} / \mathrm{PAQ}-\mathrm{A}: \text { (4 and } 5 \\
\text { points) }\end{array}$ & $10.6 \%$ & n.a. & n.a. \\
\hline Legnani et al.\#56 & $\begin{array}{c}\text { Foz do Iguaçú, } \\
\text { PR }\end{array}$ & $\geq 60 \mathrm{~min} . /$ day of MVPA & n.a. & $21.7 \% \&$ & $22.1 \% \&$ \\
\hline $\begin{array}{l}\text { Leites, Bastos and } \\
\text { Bastos }^{\# 57}\end{array}$ & Porto Alegre, RS & $\geq 300$ min./week of MVPA & n.a. & $\begin{array}{c}\text { Boys } \\
10-12 \text { years old: } \\
44.0 \% \\
13-15 \text { years old: } \\
45.6 \% \\
16-19 \text { years old: } \\
33.5 \%\end{array}$ & $\begin{array}{c}\text { Girls } \\
10-12 \text { years old: } \\
20.3 \% \\
13-15 \text { years old: } \\
20.8 \% \\
16-19 \text { years old: } \\
14.2 \%\end{array}$ \\
\hline
\end{tabular}

Continue... 


\begin{tabular}{|c|c|c|c|c|c|}
\hline \multirow{2}{*}{ Reference } & \multirow{2}{*}{ Local } & \multirow{2}{*}{ Definition } & \multicolumn{3}{|c|}{ Prevalence of Physical Activity } \\
\hline & & & All & Boys & Girls \\
\hline Lima et al. ${ }^{\text {h } 64}$ & Curitiba, PR & $\geq 60 \mathrm{~min} . /$ day of MVPA & $14.3 \%$ & $21.7 \%$ & $09.2 \%$ \\
\hline Lima and Silva ${ }^{4} 4$ & São José, SC & $\geq 60 \mathrm{~min} . /$ day of MVPA & $22.8 \%$ & $27.6 \%$ & $18.8 \%$ \\
\hline Loche\# $10^{\text {en }}$ & Santa Catarina & $\geq 300 \mathrm{~min} . /$ week of MVPA & $70.0 \%$ & $79.0 \%$ & $63.1 \%$ \\
\hline Lucena et al.g71 & João Pessoa, PB & $\geq 300 \mathrm{~min} . /$ week of MVPA & $50.2 \%$ & $66.3 \%$ & $38.5 \%$ \\
\hline Malta et al. ${ }^{d} 83$ & National & $\geq 300 \mathrm{~min} . /$ week of MVPA & $43.1 \%$ & $56,2 \%$ & $31,3 \%$ \\
\hline Malta et al. ${ }^{i 32}$ & National & $\geq 60 \mathrm{~min} . /$ day of MVPA & $\begin{array}{c}\text { Actives: } \\
\text { White }(20.5 \%) ; \\
\text { Black }(20.5 \%) \text {; Yel- } \\
\text { low }(20.1 \%) ; \text { Brown } \\
(19.6 \%) ; \text { Indigenous } \\
(22.5 \%)\end{array}$ & n.a. & n.a. \\
\hline $\begin{array}{l}\text { Martins, Lima and } \\
\text { Silva }^{\mathrm{a} 42}\end{array}$ & São José, SC & $\geq 60 \mathrm{~min} . /$ day of MVPA & $\begin{array}{l}\% \text { Actives: Poor Self- } \\
\text { rated health }(34.2 \%) \\
\text { Good Self-rated } \\
\text { health }(51.4 \%) .\end{array}$ & n.a. & n.a. \\
\hline Matsudo et al. $\$ 102$ & São Paulo & IPAQ definition & n.a. & $61.4 \%$ & $50.5 \%$ \\
\hline Meireles et al. ${ }^{91}$ & $\begin{array}{c}\text { Belo Horizonte, } \\
\text { MG }\end{array}$ & $\geq 300 \mathrm{~min} . /$ week of MVPA & $49.4 \%$ & n.a. & n.a. \\
\hline $\begin{array}{l}\text { Mendonça and } \\
\text { Farias Júniorg } 72\end{array}$ & João Pessoa, PB & $\geq 300 \mathrm{~min} . /$ week of MVPA & $50.2 \%$ & $66.3 \%$ & $38.5 \%$ \\
\hline Mendonça et al.g80 & João Pessoa, PB & $\geq 300 \mathrm{~min} . /$ week of MVPA & $50.2 \%$ & $66.3 \%$ & $38.5 \%$ \\
\hline $\begin{array}{l}\text { Menezes and } \\
\text { Duarte }^{\# 21}\end{array}$ & Sergipe & $\geq 60 \mathrm{~min} . /$ day of MVPA & $22.5 \%$ & $29.0 \%$ & $14.0 \%$ \\
\hline Moraes et al. ${ }^{\mathrm{j} \# 59}$ & Maringá, PR & $\geq 300 \mathrm{~min} . /$ week of MVPA & $43.1 \%$ & $44.3 \%$ & $42.1 \%$ \\
\hline $\begin{array}{l}\text { Moraes and } \\
\text { Falcão\# } 58\end{array}$ & Maringá, PR & $\geq 60 \mathrm{~min} . /$ day of MVPA & n.a. & $44.0 \%$ & $42.1 \%$ \\
\hline Nakamura et al. ${ }^{92}$ & Rio Claro, SP & $\begin{array}{c}\text { PAQ-C / PAQ-A: (4 and } 5 \\
\text { points) }\end{array}$ & $17.4 \%$ & $26.8 \%$ & $09.4 \%$ \\
\hline Nascente et al. ${ }^{22}$ & Goiânia, G0 & $\geq 300 \mathrm{~min} . /$ week of MVPA & $33.2 \%$ & n.a. & n.a. \\
\hline $\begin{array}{l}\text { Nascimento- } \\
\text { Ferreira et al. }{ }^{103}\end{array}$ & Imperatriz, MA & $\geq 60 \mathrm{~min} . /$ day of MVPA & $45.6 \%$ & $42.7 \%$ & $69.2 \%$ \\
\hline Nunes et al. ${ }^{\text {a } 43}$ & São José, SC & $\geq 60 \mathrm{~min} . /$ day of MVPA & $22.7 \%$ & $27.7 \%$ & $18.7 \%$ \\
\hline $\begin{array}{l}\text { Pelegrini and } \\
\text { Petroskik } 60^{\text {Petro }}\end{array}$ & Florianópolis, SC & $\geq 300 \mathrm{~min} . /$ week of MVPA & n.a. & $\begin{array}{c}\text { Actives: } \\
14 \text { years }=78.6 \% ; \\
15 \text { years }=67.3 \% ; \\
16 \text { years }=84.8 \% ; \\
17 \text { years }=77.2 \% ; \\
18 \text { years }=90.0 \%\end{array}$ & $\begin{array}{c}\text { Ativas: } \\
14 \text { years }=57.1 \% ; \\
15 \text { years }=76.4 \% ; \\
16 \text { years }=76.2 \% ; \\
17 \text { years }=71.6 \% ; \\
18 \text { years }=59.1 \%\end{array}$ \\
\hline $\begin{array}{l}\text { Pereira, Bergmann } \\
\text { and Bergmann }{ }^{\mathrm{b} \$ 17}\end{array}$ & Uruguaiana, RS & $\begin{array}{c}\text { PAQ-C / PAQ-A: (4 and } 5 \\
\text { points) }\end{array}$ & $08.3 \%$ & n.a. & n.a. \\
\hline Peres et al. ${ }^{193}$ & Piracicaba, SP & $\geq 300 \mathrm{~min} . /$ week of MVPA & n.a. & $\begin{array}{c}\text { Overweight: } \\
\text { 92.5\%; Eutrophic: } \\
93.1 \%\end{array}$ & $\begin{array}{c}\text { Overweight: } \\
\text { 78,9\%; Eutrophic: } \\
84,1 \%\end{array}$ \\
\hline Petribú et al. ${ }^{\mathrm{m} 74}$ & Caruaru, PE & $\geq 60 \mathrm{~min} . /$ day of MVPA & $\begin{array}{l}\text { Overweight individu- } \\
\text { als: } 15.6 \% \\
\text { Obese individuals: } \\
3.8 \%\end{array}$ & n.a. & n.a. \\
\hline Prado et al. ${ }^{\text {h } 53}$ & Curitiba, PR & $\geq 60 \mathrm{~min} . /$ day of MVPA & $14.5 \%$ & $22.2 \%$ & $09.2 \%$ \\
\hline Quadros et al.\#23 & Amargosa, BA & $\begin{array}{l}16,000 \text { and } 13,000 \text { steps/ } \\
\text { day for boys and girls } \\
\text { (respectively). }\end{array}$ & $35.7 \%$ & n.a. & n.a. \\
\hline Raizel et al.\# 24 & Cuiabá, MT & $\geq 60 \mathrm{~min} . /$ day of MVPA & $18.0 \%$ & $31.0 \%$ * & $09.0 \%{ }^{*}$ \\
\hline
\end{tabular}

Continue.. 
.. continue

\begin{tabular}{|c|c|c|c|c|c|}
\hline \multirow{2}{*}{ Reference } & \multirow{2}{*}{ Local } & \multirow{2}{*}{ Definition } & \multicolumn{3}{|c|}{ Prevalence of Physical Activity } \\
\hline & & & All & Boys & Girls \\
\hline Regis et al. ${ }^{\text {25 }}$ & Pernambuco & $\geq 60 \mathrm{~min} . /$ day of MVPA & $\begin{array}{l}\text { Urban area: } 34.5 \% \\
\text { Rural area: } 37.3 \%\end{array}$ & n.a. & n.a. \\
\hline Reichert et al. ${ }^{61}$ & Pelotas, RS & $\geq 60 \mathrm{~min} . /$ day of MVPA & $61.3 \%$ & $69.8 \%$ & $52.1 \%$ \\
\hline Reis et al. ${ }^{n}{ }^{54}$ & Curitiba, PR & $\geq 60 \mathrm{~min} . /$ day of MVPA & n.a. & $21.7 \%$ & $09.1 \%$ \\
\hline Rezende et al. ${ }^{i 33}$ & Nacional & $\geq 60 \mathrm{~min} . /$ day of MVPA & $29.0 \%$ & $38.6 \%$ & $20.1 \%$ \\
\hline Rivera et al. ${ }^{0 * 12}$ & Maceió, AL & $\geq 60 \mathrm{~min} . /$ day of MVPA & $06.5 \%$ & n.a. & n.a. \\
\hline Rodrigues et al. ${ }^{81}$ & Cuiabá, MT & $\geq 300$ min./week of MVPA & $40.5 \%$ & n.a. & n.a. \\
\hline Romanzini et al. ${ }^{\# 55}$ & Londrina, PR & $\geq 300$ min./week of MVPA & $60.8 \%$ & $66.7 \%$ & $57.2 \%$ \\
\hline Romero et al. ${ }^{94}$ & Piracicaba, SP & $\geq 300$ min./week of MVPA & $45.1 \%^{\#}$ & n.a. & n.a. \\
\hline $\begin{array}{l}\text { Salvador, Kitoko } \\
\text { and Gambardella }{ }^{95}\end{array}$ & Vitória, ES & $5+$ hours per week of PA & $\begin{array}{c}\text { Normal weight: } \\
79.2 \% ; \\
\text { overweight: } 20.8 \%\end{array}$ & n.a. & n.a. \\
\hline Santos et al. ${ }^{\mathrm{h} 51}$ & Curitiba, PR & $\geq 60 \mathrm{~min} . /$ day of MVPA & n.a. & $22.0 \%$ & $09.1 \%$ \\
\hline Santos et al. ${ }^{35}$ & Uberaba, MG & $\geq 60 \mathrm{~min} . /$ day of MVPA & $55.1 \%$ & n.a. & n.a. \\
\hline Santos et al. ${ }^{n 96}$ & Pernambuco & $\geq 60 \mathrm{~min} . /$ day of MVPA & $35.1 \%$ & $43.9 \%$ & $29.2 \%$ \\
\hline Santos et al. ${ }^{c 31}$ & Pernambuco & $\geq 60 \mathrm{~min} . /$ day of MVPA & $34.9 \%{ }^{\#}$ & $42.3 \%$ & $29.8 \%$ \\
\hline Silvai 34 & National & $\geq 60 \mathrm{~min} . /$ day of MVPA & n.a. & $27.9 \%$ & $13.1 \%$ \\
\hline Silva and Silva ${ }^{p 28}$ & Aracajú, SE & $\geq 60 \mathrm{~min} . /$ day of MVPA & $18.1 \%$ & $26.8 \%$ & $12.9 \%$ \\
\hline Silva et al. .\#13 & Maceió, AL & $\begin{array}{c}\text { PAQ-C / PAQ-A: (3 to } 5 \\
\text { points) }\end{array}$ & $06.5 \%$ & n.a. & n.a. \\
\hline Silva et al. ${ }^{e \# 8}$ & Santa Catarina & $\geq 300$ min./week of MVPA & n.a. & $78.9 \%$ & $63.0 \%$ \\
\hline Silva et al. ${ }^{97}$ & Aracajú, SE & $\begin{array}{c}P A Q-C \text { / PAQ-A: (4 and } 5 \\
\text { points) }\end{array}$ & n.a. & $2.0 \%$ & $0.3 \%$ \\
\hline Silva et al. ${ }^{e 9}$ & Santa Catarina & $\geq 300 \mathrm{~min}$./week of MVPA & $71.5 \%$ & $72.1 \%^{\#}$ & $71.2 \%{ }^{*}$ \\
\hline Silva et al. ${ }^{p} 18$ & Aracajú, SE & $\geq 60 \mathrm{~min} . /$ day of MVPA & n.a. & $27.2 \%$ & $12.6 \%$ \\
\hline Silva et al. ${ }^{27}$ & Santa Catarina & $\geq 300$ min./week of MVPA & n.a. & $39.3 \%$ & $23.7 \%$ \\
\hline Silva et al. ${ }^{64}$ & $\begin{array}{c}\text { Ponta Grossa, } \\
\text { PR }\end{array}$ & $\geq 300 \mathrm{~min}$./week of MVPA & $92.3 \%$ & n.a. & n.a. \\
\hline Silva et al. ${ }^{k 65}$ & Florianópolis, SC & $\geq 300$ min./week of MVPA & $68.2 \%$ & $79.6 \%$ & $71.6 \%$ \\
\hline Silva et al. $\$ 15$ & Arapiraca, SE & $\begin{array}{l}37 \text { or more points in the } \\
\text { instrument score. }\end{array}$ & $10.9 \%{ }^{\#}$ & n.a. & n.a. \\
\hline $\begin{array}{l}\text { Silva, Silva and } \\
\text { Oliveira }{ }^{\dagger} 18\end{array}$ & Aracajú, SE & $\begin{array}{c}\text { PAQ-C / PAQ-A: (3 to } 5 \\
\text { points) }\end{array}$ & n.a. & $20.6 \%$ & $04.8 \%$ \\
\hline $\begin{array}{l}\text { Silva, Smith-Men- } \\
\text { ezes and Duarte }\end{array}$ & Sergipe & $\geq 300$ min./week of MVPA & $22.5 \%$ & $18.4 \%$ & $29.0 \%$ \\
\hline Souza ${ }^{98}$ & Canoas, RS & $\geq 300$ min./week of MVPA & $47.7 \%$ & n.a. & n.a. \\
\hline Souza et al. $\# 76$ & Salvador, BA & $\geq 300$ min./week of MVPA & n.a. & $72.0 \%$ & $50.0 \%$ \\
\hline Souza et al." 52 & Curitiba, PR & $\geq 60 \mathrm{~min} . /$ day of MVPA & $14.5 \%$ & $21.6 \%$ & $09.6 \%$ \\
\hline $\begin{array}{l}\text { Straatmann and } \\
\text { Veiga }^{99}\end{array}$ & Niterói, RJ & $\begin{array}{c}\text { IPAQ short version: } \\
\text { active= very active and } \\
\text { active }\end{array}$ & n.a. & $69.1 \%$ & $54.7 \%$ \\
\hline Suñé et al. $\$ 62$ & $\begin{array}{c}\text { Capão da Canoa, } \\
\text { RS }\end{array}$ & $\begin{array}{c}6 \text { or more points in instru- } \\
\text { ment score. }\end{array}$ & $21.2 \%$ & n.a. & n.a. \\
\hline Tassitano et al. ${ }^{\mathrm{m} \# 75}$ & Caruaru, PE & $\geq 60 \mathrm{~min} . /$ day of MVPA & $58.3 \%$ & $77.2 \%$ & $46.9 \%$ \\
\hline Tenório et al. ${ }^{c * 68}$ & Pernambuco & $\geq 60 \mathrm{~min} . /$ day of MVPA & $34.9 \%$ & $42.3 \%$ & $29.8 \%$ \\
\hline Vieira et al..$^{100}$ & Natal, RN & $\geq 300$ min./week of MVPA & $\begin{array}{c}\text { Normal weight: } \\
46.9 \% \text {. } \\
\text { overweight: } \\
50.0 \%\end{array}$ & n.a. & n.a. \\
\hline
\end{tabular}

Continue... 


\begin{tabular}{|c|c|c|c|c|c|}
\hline \multirow{2}{*}{ Reference } & \multirow{2}{*}{ Local } & \multirow{2}{*}{ Definition } & \multicolumn{3}{|c|}{ Prevalence of Physical Activity } \\
\hline & & & All & Boys & Girls \\
\hline Vitta et al. ${ }^{101}$ & Bauru, SP & $\begin{array}{c}P A Q-C \text { / PAQ-A: (3 to } 5 \\
\text { points) }\end{array}$ & $23.5 \%$ & $44.6 \%$ & $18.7 \%$ \\
\hline
\end{tabular}

Note. States of Brazil: AL: Alagoas; BA: Bahia; DF: Distrito Federal; ES: Espírito Santo; GO: Goiás; MA: Maranhão; MG: Minas Gerais; MT: Mato Grosso; PB: Paraíba; PE: Pernambuco; PI: Piauí; PR: Paraná; RJ: Rio de Janeiro; RN: Rio Grande do Norte; RS: Rio Grande do Sul; SC: Santa Catarina; SE: Sergipe; SP: São Paulo n.a.: not avaliable. a - Brazilian Guide for Evaluation of Health-Related Physical Fitness and Life Habits - Stage I; b - Estudo em Uruguaiana/RS - protocol do Comitê de Ética em Pesquisa: 042/2010; c - Estilos de Vida e Comportamentos de Risco à Saúde em Estudantes do Ensino Médio no Estado de Pernambuco; d - Pesquisa Nacional de Saúde do Escolar, edição 2009 - PeNSE 2009; e - Estilo de Vida e Comportamentos de Risco dos Jovens Catarinenses, edição 2001 - COMPAC 2001; $f$-Estudo em Sergipe - protocolo do Comitê de Ética em Pesquisa da Universidade Federal de Sergipe (CEP/UFS): $5724.0 .000 .107-$ 10.; $g$ - Physical activity level and associated factors among high school adolescents in João Pessoa, city, - PB: an ecological approach.; h - Determinantes da Atividade Física e Obesidade em Escolares do Ensino Médio da Rede Pública da Cidade de Curitiba, Paraná, Brasil.; i - Pesquisa Nacional de Saúde do Escolar, edição 2012 - PeNSE 2012; j - Estudo em Maringá/PR - Aprovado pelo Comitê de Ética em Pesquisa com Seres Humanos do Centro Universitário de Maringá.; $k$ - Níveis de atividade física, aptidão física e comportamento social relacionados à saúde em escolares de Florianópolis, SC.; I - Consumo dietético e AF como determinantes das mudanças do IMC de uma coorte de adolescentes matriculados da rede pública de ensino da cidade de Piracicaba/SP.; m - Estudo em Caruaru/PE - protocolo do Comitê de Ética em Pesquisa com Seres Humanos da Faculdades ASCES: 44/2007.; n - Physical activity practice and health risk behaviors in high school students in the State of Pernambuco: a temporal trend study.; 0 - Estudo em Maceió-AL - Aprovado pelo Comitê de Ética do Hospital Universitário da Universidade Federal de Alagoas.; $p$ - Estudo em Aracajú/SE - Aprovado pelo Ethics Committee on Human Research of the Federal University of Sergipe. ${ }^{\$} \%$ very active + \%active; ${ }^{\#}$ Calculated prevalences of physical activity: $100 \%$ - \% of physical inactivity (original information provided in the articles); ${ }^{\circ}$ Data from brazilian adolecents; "Scores 3, 4 and 5 summed.

\section{DISCUSSION}

The objective of this systematic review was to synthesize the studies on the prevalence of physical activity in children and adolescents in Brazil. Our findings showed that there was a wide range between studies in the prevalence of physically active children and adolescents. However, thirty-five studies found a prevalence of physically active children and adolescents lower than $50 \%{ }^{12,13,23-31,36,14,40-43,45,49-53,16,54,56-59,62,63,66-68,17,81,90-92,96-98,101,18-22}$, including two National School Health Surveys ${ }^{33,84}$. Thus, it seems that increasing physical activity practices among Brazilian children and adolescents is a priority.

The large variability between studies in prevalence rates may be explained by the distinctions in the instruments/questionnaires used. Recording period, instrument structure, wording of questionnaires, physical activity domains included, and cutoff points were aspects that varied between surveys (Table 1). For example, Farias Júnior et al. ${ }^{73}$ found that estimates of physically active adolescents using simplified and detailed instruments, can have a relative difference of $100 \%$ or higher. Subjective measures of physical activity are still predominantly used in epidemiological research in Brazil ${ }^{104}$ and other low- and middle-income countries due to the context of where the data is recorded and the low operational costs involved $^{105}$. However, it is important that future studies consider developing and using validated and reliable instruments for physical activity measurement in epidemiological surveys in Brazil.

Inversely, this systematic review included only two studies with objectively-measured physical activity ${ }^{23,61}$. In Brazil, the high cost of objective measures of physical activity can still be a barrier to their use in comprehensive survey studies. Another obstacle to be overcome for optimizing the use of objective instruments, in particular accelerometers, is the problematic comparability between different models and outputs ${ }^{106}$. Comprehensive 
surveys using objectively-measured physical activity (e.g., accelerometers) are still on the rise in Brazil, and well-designed studies should be developed in order to reduce comparability issues of physical activity data.

Most of studies in Brazil highlighted physical activity distinctions for boys and girls, which was similar to the global estimate ${ }^{107}$. However, when considering age groups, no studies were found that investigated the prevalence of physically active preschool children (under 6 years old). An explanation for this result may be that the measurement of physical activity with subjective instruments is unreliable and most of questionnaires were not validated for younger children.

The results of this systematic review highlighted other trends in the research on physical activity prevalence in Brazil. Since 2001, there has been an increase in research on physical activity in children and adolescents with a concomitant evolution of the robustness of the studies developed in the Brazilian scientific community. This is also supported by two versions of the National Survey of School Health (2009 and 2012), which provided information for the Surveillance System for Risk Factors for Non-communicable Chronic Diseases ${ }^{34,84}$. These results reflect the emphasis on epidemiological surveys in the Brazilian physical activity research agenda during this period.

The results showed that most of the studies were carried out in South and Northeast regions of Brazil. Previous systematic reviews ${ }^{104,108}$ found a large number of studies on physical activity and health in the South and Southeast regions of Brazil. Thus, a positive finding in the current review was the relevant number of included studies in Northeastern Brazil. This may be related to the number of research groups and graduate programs in Physical Education in this region of Brazil, which can stimulate new and well-designed research practices for studies in these areas. However, the development of studies in the Northern region of Brazil is urgent, because no studies of this region were included in the current review and physical activity promotion strategies for young populations in the Northern area need to consider context-specific needs and peculiarities. Research groups focused on physical activity and health should be stimulated and integrated with other multicenter studies to promote best research practice for studies on physical activity and health in this region.

This systematic review is important because research on the physical activity level of Brazilian children and adolescents was presented with new evidence in comparison to previous reviews of this topic ${ }^{109}$. Also, gaps and recommendations for studying physical activity were highlighted. However, a large variability in the measurement of physical activity between studies limited the possibilities of a quantitative synthesis (meta-analysis) of the physical activity prevalence rates from all studies and of a discussion of other relevant aspects of physical activity in Brazil, such as secular trends.

\section{CONCLUSION}

The current review found that there was great variability in the measure- 
ment of physical activity and cut-off points among the studies with children and adolescents in Brazil. The large range of physical activity prevalence was something remarkable in this review. However, 35 studies found a prevalence of physically active children and adolescents lower than 50\%. Thus, it seems that physical activity practices among Brazilian children and adolescents is still a challenge for the country. Important research gaps (e.g., studies with objectively-measured physical activity, children aged up to 6 years old and in Northern Brazil) should be considered when developing new research studies in Brazil.

\section{COMPLIANCE WITH ETHICAL STANDARDS}

\section{Funding}

This research did not receive any specific grant from funding agencies in the public, commercial, or not-for-profit sectors. This study was funded by the authors.

\section{Conflict of interest statement}

The authors have no conflict of interests to declare.

\section{Author Contributions}

VCBF participated in the methodological design, search and evaluation of studies, writing the text and approved the final version of the manuscript. RMC, MCTGK, BNO and CBA participated in the methodological design, study search and selection, data extraction and synthesis, writing the text and approved the final version of the manuscript. KSS participated in the methodological design, evaluation of studies, writing the text and approved the final version of the manuscript.

\section{REFERENCES}

1. Committee 2018 Physical Activity Guidelines Advisory. 2018 Physical Activity Guidelines Advisory Committee Scientific Report. 2018. 779 p.

2. Hallal PC, Andersen LB, Bull FC, Guthold R, Haskell W, Ekelund U, et al. Global physical activity levels: Surveillance progress, pitfalls, and prospects. Lancet 2012;380(9838):247-57.

3. Malta DC, Andreazzi MAR, Oliveira-Campos M, Andrade SSCA, Sá NNB, Moura L, et al. Trend of the risk and protective factors of chronic diseases in adolescents, National Adolescent School-based Health Survey (PeNSE 2009 e 2012). Rev Bras Epidemiol 2014;17(suppl 1):77-91.

4. The Active Healthy Kids Global Alliance. The Global Matrix 3.0 on Physical Activity for Children and Youth. 2018. Available from: https://www.activehealthykids. org/. Accessed in: 01 May, 2018.

5. Nardo Júnior N, Silva DAS, Ferrari GLM, Petroski EL, Pacheco RL, Martins PC, et al. Results From Brazil's 2016 Report Card on Physical Activity for Children and Youth. J Phys Act Health 2016;13(Suppl 2r):104-9.

6. Caspersen CJ, Powell KE, Christenson GM. Physical activity, exercise, and physical fitness: definitions and distinctions for health-related research. Public Health Rep 1985;100(2):126-31.

7. World Health Organization. Global recommendations on physical activity for health. Geneva: WHO, 2010. 
8. Silva KS, Nahas MV, Hoefelmann LP, Lopes AS, Oliveira ES. Associações entre atividade física, índice de massa corporal e comportamentos sedentários em adolescentes. Rev Bras Epidemiol 2008;11(1):159-68.

9. Silva K, Nahas M, Peres K, Lopes A. Fatores associados à atividade física, comportamento sedentário e participação na Educação Física em estudantes do Ensino Médio em Santa Catarina, Brasil. Cad Saúde Pública 2009;25(10):2187-200.

10. Loch M. Indicadores de saúde e do estilo de vida de adolescentes escolares residentes em municípios grandes, médios e pequenos de Santa Catarina, Brasil. Rev Bras Ciência Mov 2007;15(3):7-14.

11. Farias Júnior JC, Nahas MV, Barros MVG, Loch MR, Oliveira ES, De Bem MFL, et al. Comportamentos de risco à saúde em adolescentes no Sul do Brasil: prevalência e fatores associados. Rev Panam Salud Pública 2009;25(4):344-52.

12. Rivera IR, da Silva MAM, Silva RTA, Oliveira BAV, Carvalho ACC. Physical inactivity, TV-watching hours and body composition in children and adolescents. Arq Bras Cardio. 2010;95(2):159-65.

13. Silva M, Rivera I, Ferraz M, Pinheiro A, Alves S. Prevalência de fatores de risco cardiovascular em crianças e adolescentes da rede de ensino da cidade de Maceió. Arq Bras Cardiol 2005;84(5):387-92.

14. Farias Júnior JC. Prevalência e fatores de influência para inatividade física em adolescentes. Rev Bras Ciênc Mov 2006;14(2):57-64.

15. Silva LCB, Tassitano RM, Medeiros HJ, Knackfuss MI, Cunha Júnior AT. Excesso de peso e fatores comportamentais. Motri 2016;12(Suppl. 2):112-23.

16. Bergmann GG, Bergmann MLA, Marques AC, Hallal PC. Prevalence of physical inactivity and associated factors among adolescents from public schools in Uruguaiana, Rio Grande do Sul State, Brazil. Cad Saúde Pública 2013;29(11):2217-29.

17. Pereira TA, Bergmann MLA, Bergmann GG. Fatores associados à baixa aptidão física de adolescentes. Rev Bras Med Esporte 2016;22(3):176-81.

18. Silva RJS, Silva DAS, Oliveira AC. Low physical activity levels and associated factors in Brazilian adolescents from public high schools. J Phys Act Health 2014;11(7):1438-45.

19. Leão AS, Moura Soares NM, Gonçalves ECDA, Silva DAS, Silva RJS, Thomazzi SM. Simultaneous health risk behaviors in adolescents associated with higher economic class in the Northeast of Brazil. Sci World J 2017;2017:1-7.

20. Dumith SC, Santos MN, Teixeira LO, Cazeiro CC, Mazza SEI, Cesar JA. Prática de atividade física entre jovens em município do semiárido no Brasil. Cien Saude Colet 2016;21(4):1083-93.

21. Menezes AS, Duarte MFS. Condições de vida, inatividade física e conduta sedentária de jovens nas áreas urbana e rural. Rev Bras Med Esporte 2015;21(5):338-44.

22. Nascente FMN, Jardim TV, Peixoto MRG, Carneiro CS, Mendonça KL, Póvoa TIR, et al. Sedentary lifestyle and its associated factors among adolescents from public and private schools of a Brazilian state capital. BMC Public Health 2016;16:1177.

23. Quadros TMB, Gordia AP, Silva LR, Silva DAS, Mota J. Inquérito epidemiológico em escolares: determinantes e prevalência de fatores de risco cardiovascular. Cad Saude Publica 2016;32(2): e00181514.

24. Raizel R, Silva VG, Godois AM, Martínez Espinosa M, Dreyer Machado A, Júnior Henrique Duarte $\mathrm{S}$, et al. Comportamentos de risco à saúde de adolescentes e atividades educativas da Estratégia Saúde da Família em Cuiabá, Mato Grosso, 2011. Epidemiol Serv Saúde 2016;25(2): 291-9.

25. Regis MF, Oliveira LMFT, Santos ARM, Leonidio ACR, Diniz PRB, Freitas CMSM. Urban versus rural lifestyle in adolescents: associations between environment, physical activity levels and sedentary behavior. Einstein 2016;14(4):461-7.

26. Cureau FV, Duarte P, Santos DL, Reichert FF. Clustering of risk factors for noncommunicable diseases in Brazilian adolescents: Prevalence and correlates. J Phys Act Health 2014;11(5):942-9. 
27. Silva KS, Barbosa Filho VC, Del Duca GF, Peres MAA, Mota J, Lopes AS, Nahas MV. Gender differences in the clustering patterns of risk behaviours associated with non-communicable diseases in Brazilian adolescents. Prev Med 2014;65(8):77-81

28. Silva DAS, Silva RJS. Association between physical activity level and consumption of fruit and vegetables among adolescents in northeast Brazil. Rev Paul Pediatr 2015;33(2):167-73.

29. Silva FMA, Smith-Menezes A, Duarte MFS. Consumo de frutas e vegetais associado a outros comportamentos de risco em adolescentes no Nordeste do Brasil. Rev Paul Pediatr 2016;34(3):309-15.

30. Silva DAS, Tremblay MS, Gonçalves ECDA, Silva RJDS. Television time among Brazilian adolescents: Correlated factors are different between boys and girls. Sci World J 2014;2014:794539.

31. Santos SJ, Hardman CM, Barros SSH, Barros MVG. Association between physical activity, participation in Physical Education classes, and social isolation in adolescents. J Pediatr 2015;91(6):543-50.

32. Malta DC, Stopa SR, Santos MAS, Andrade SSCA, Oliveira MM, Prado RR, et al. Fatores de risco e proteção de doenças e agravos não transmissíveis em adolescentes segundo raça/cor: Pesquisa Nacional de Saúde do Escolar. Rev Bras Epidemiol 2017;20(2):247-59.

33. Rezende LFM, Azeredo CM, Canella DS, Claro RM, Castro IRR, Bertazzi LR, et al. Sociodemographic and behavioral factors associated with physical activity in Brazilian adolescents. Rev Paul Pediatr 2014;32(3):241-6.

34. Silva DAS. Relationship between Brazilian adolescents' physical activity and social and economic indicators of the cities where they live. Percept Mot Skills 2015;120(2):355-66.

35. Santos A, Andaki ACR, Amorim PRS, Mendes EL. Fatores associados ao comportamento sedentário em escolares de 9-12 anos de idade. Motriz: J Phys Ed 2013;19(Suppl. 3):25-34.

36. Ceschini FL, Andrade EL, Figueira Júnior A. Physical activity and associated factors among students attending evening classes. Rev Bras Cineantropom Desempenho Hum 2015;17(2):205-15.

37. Guilherme FR, Molena-Fernandes CA, Guilherme VR, Fávero MTM, Reis EJB, Rinaldi W. Inatividade física e medidas antropométricas em escolares de Parnavaí, Paraná, Brasil. Rev Paul Pediatr 2015;33(1):50-5.

38. Vieira CENK, Enders BC, Coura AS, Lira ALBC, Medeiros CCM, Mariz LC. Nursing diagnosis of overweight and related factors in adolescents. Investig Educ Enfermería 2015;33(3):509-18.

39. Nascimento-Ferreira MV, Moraes ACF, Rendo-Urteaga T, Oliveira Forkert EC, Collese TS, Cucato GG, et al. Cross-sectional, school-based study of 14-19 year olds showed that raised blood pressure was associated with obesity and abdominal obesity. Acta Paediatr 2017;106(3):489-96.

40. Alves Junior CAS, Gonçalves ECA, Silva DAS. Obesity in adolescents in Southern Brazil: association with sociodemographic factors, lifestyle and maturational stage. Rev Bras Cineantropom Desempenho Hum 2016;18(5):557-66.

41. Lima TR, Silva DAS. Prevalence of physical activity among adolescents in southern Brazil. J Body Mov Ther 2018;22(1):57-63.

42. Martins PC, Lima TR, Silva DAS. Association between different contexts of physical activity and abdominal obesity and excess weight in adolescents. Motriz: J Phys Ed 2018;23(Suppl.2):1017104.

43. Nunes HEG, Gonçalves ECDA, Vieira JAJ, Silva DAS. Clustering of risk factors for non-communicable diseases among adolescents from southern Brazil. PLoS One 2016;11(7):0159037.

44. Arruda ELM, Lopes A. Gordura corporal, nível de atividade física e hábitos alimentares de adolescentes da região serrana de Santa Catarina, Brasil. Rev Bras Cineantropom Desempenho Hum 2007;9(1):5-11. 
45. Beck CC, Lopes AS, Giuliano ICB, Borgatto AF. Fatores de risco cardiovascular em adolescentes de município do sul do Brasil: prevalência e associações com variáveis sociodemográficas. Rev Bras Epidemiol 2011;14(1):36-49.

46. Campos W, Stabelini Neto A, Bozza R, Ulbrich AZ, Bertin RL, Mascarenhas $\mathrm{LPG}$, et al. Atividade física, consumo de lipídios e fatores de risco para aterosclerose em adolescentes. Arq Bras Cardiol 2010;94(5):601-7.

47. Dambros DD, Lopes LFD, Santos DL. Barreiras percebidas e hábitos de atividade física de adolescentes escolares de uma cidade do sul do brasil. Rev Bras Cineantropom Desempenho Hum 2011;13(6):422-8.

48. Souza RP. Os beneficios da prática de atividade física e os riscos do sedentarismo em: crianças e adolescentes, no adulto e no idoso. Cinergis 2010;11(1):52-9.

49. Fermino RC, Rech CR, Hino AAF, Rodriguez Añez CR, Reis RS, RodriguezAñez CR, et al. Atividade física e fatores associados em adolescentes do ensino médio de Curitiba, Brasil. Rev Saúde Pública 2010;44(6):986-95.

50. Lima AV, Fermino RC, Oliveira MP, Rodriguez Añez CR, Reis RS. Distância percebida até as instalações de lazer e sua associação com a prática de atividade física e de exercícios em adolescentes de Curitiba, Paraná, Brasil. Cad Saude Publica 2013;29(8):1507-21.

51. Santos MS, Hino AAF, Reis R, Rodriguez-Añez C. Prevalência de barreiras para a prática de atividade física em adolescentes. Rev Bras Epidemiol 2010;13(1):94-104.

52. Souza CA, Rech CR, Sarabia TT, Añez CRR, Reis RS. Autoeficácia e atividade física em adolescentes de Curitiba, Paraná, Brasil. Cad Saude Publica 2013;29(10):2039-48.

53. Prado CV, Lima AV, Fermino RC, Añez CRR, Reis RS. Apoio social e prática de atividade física em adolescentes da rede pública de ensino: qual a importância da família e dos amigos? Cad Saude Publica 2014;30(4):827-38.

54. Reis RS, Hino AAF, Florindo AA, Anez CRR, Domingues MR. Association between physical activity in parks and perceived environment: a study with adolescents. J Phys Act Health 2009;6(4):503-9.

55. Romanzini M, Reichert FF, Lopes AS, Petroski EL, Farias Júnior JC. Prevalência de fatores de risco cardiovascular em adolescentes. Cad Saúde Pública 2008;24(11):2573-81.

56. Legnani E, Legnani RFS, Barbosa Filho VC, Gasparotto GS, Campos W, Lopes AS. Fatores de risco à saúde cardiovascular em escolaresda Tríplice Fronteira. Motriz: J Phys Ed 2011;17(4):640-9.

57. Leites GT, Bastos GAN, Bastos JP. Prevalence of insufficient physical activity in adolescents in South Brazil. Rev Bras Cineantropom Desempenho Hum 2013;15(3):286-95.

58. Moraes ACF, Falcão MC. Lifestyle factors and socioeconomic variables associated with abdominal obesity in Brazilian adolescents. Ann Hum Biol 2013;40(1):1-8.

59. Moraes ACF, Fernandes CAM, Elias RGM, Nakashima ATA, Reichert FF, Falcão MC. Prevalência de inatividade física e fatores associados em adolescentes. Rev Assoc Med Bras 2009;55(5):523-8.

60. Pelegrini A, Petroski EL. Inatividade física e sua associação com estado nutricional, insatisfação com a imagem corporal e comportamentos sedentários em adolescentes de escolas públicas. Rev Paul Pediatr 2009;27(4):366-73.

61. Reichert FF, Menezes AMB, Hallal PC, Ekelund U, Wells JCK. Objectively measured physical activity and body composition indices in Brazilian adolescents. Rev Bras Ativ Fis Saude 2012;17(6):573-84.

62. Suñé FR, Dias-da-Costa JS, Olinto MTA, Pattussi MP. Prevalência e fatores associados para sobrepeso e obesidade em escolares de uma cidade no Sul do Brasil. Cad Saude Publica 2007;23(6):1361-71.

63. Bastos JP, Araújo CLP, Hallal PC. Prevalence of insufficient physical activity and associated factors in Brazilian adolescents. J Phys Act Health 2008;5(6):777-94.

64. Silva DAS, Lima LRA, Dellagrana RA, Bacil EDA, Rech CR. Pressão arterial elevada em adolescentes: prevalência e fatores associados. Cien Saude Colet 2013;18(11):3391-400. 
65. Silva DAS, Tremblay MS, Pelegrini A, Silva JMFL, Petroski EL. Low aerobic fitness in Brazilian adolescents. Rev Bras Med Esporte 2015;21(2):94-8.

66. Barros MVG, Ritti-Dias RM, Barros SSH, Mota J, Andersen LB. Does selfreported physical activity associate with high blood pressure in adolescents when adiposity is adjusted for? J Sports Sci 2013;31(4):387-95.

67. Cavalcanti CBS, Barros MVG, Menêses AL, Santos CM, Azevedo AMP, Guimarães FJSP. Obesidade Abdominal em Adolescentes: Prevalência e Associação com Atividade Física e Hábitos Alimentares. Arq Bras Cardiol 2010;94(3):371-7.

68. Tenório MCM, Barros MVG, Tassitano RM, Bezerra J, Tenório JM, Hallal PC. Atividade física e comportamento sedentário em adolescentes estudantes do ensino médio. Rev Bras Epidemiol 2010;13(1):105-17.

69. Farias Júnior JC, Lopes AS, Mota J, Hallal PC. Prática de atividade física e fatores associados em adolescentes no Nordeste do Brasil. Rev Saude Publica 2012;46(3):505-15.

70. Farias Junior JC, Reis RS, Hallal PC. Physical activity, psychosocial and perceived environmental factors in adolescents from Northeast Brazil. Cad Saúde Pública 2014;30(5):941-51.

71. De Lucena JMS, Cheng LA, Cavalcante TLM, Silva VA, Farias Júnior JC. Prevalence of excessive screen time and associated factors in adolescents. Rev Paul Pediatr 2015;33(4):407-14.

72. Mendonça G, Farias Júnior JC. Physical activity and social support in adolescents: analysis of different types and sources of social support. J Sports Sci 2015;33(18):1942-51.

73. Farias Júnior JC, Barbosa AO, Mendes JKF, Mendonça G. Prevalence of sufficient levels of physical activity in adolescents: comparison between the measure obtained using simplified and detailed questionnaires. Rev Bras Cineantropom Hum 2016;18(4):450-9.

74. Petribú MMV, Tassitano RM, Nascimento WMF, Santos EMC, Cabral PC. Fatores associados ao sobrepeso e à obesidade em estudantes do ensino médio da rede pública estadual do município de Caruaru (PE). Rev Paul Pediatr 2011;29(4):536-45.

75. Tassitano RM, Dumith SC, Chica DAG, Tenório MCM. Agregamento dos quatro principais comportamentos de risco às doenças não transmissíveis entre adolescentes. Rev Bras Epidemiol 2014; 17(2):465-78.

76. Souza CO, Silva RCR, Assis AMO, Fiaccone RL, Pinto EDJ, Moraes LTLP. Associação entre inatividade física e excesso de peso em adolescentes de Salvador, Bahia-Brasil. Rev Bras Epidemiol 2010;13(3):468-75.

77. Carvalho RBN, Nobre RS, Guimarães MR, Teixeira SEXM, Silva ARV. Fatores de risco associados ao desenvolvimento da síndrome metabólica em crianças e adolescentes. Acta Paul Enferm 2016;29(4):439-45.

78. Farias Júnior JC. Associação entre prevalência de inatividade física e indicadores de condição socioeconômica em adolescentes. Rev Bras Med Esporte 2008;14(2):109-14.

79. Farias Júnior JC, Lopes AS, Mota J, Santos MP, Ribeiro JC, Hallal PC. Perception of the social and built environment and physical activity among Northeastern Brazil adolescents. Prev Med 2011;52(2):114-9.

80. Mendonça G, Florindo AA, Rech CR, Freitas DKS, Farias Júnior JC. Perceived neighborhood environmental characteristics and different types of physical activity among Brazilian adolescents. J Sports Sci 2017;36(9):1068-1075.

81. Rodrigues PRM, Pereira RA, Cunha DB, Sichieri R, Ferreira MG, Vilela AAF, et al. Fatores associados a padrões alimentares em adolescentes: um estudo de base escolar em Cuiabá, Mato Grosso. Rev Bras Epidemiol 2012;15(3):662-74.

82. Abreu MNS, Caiaffa WT. Influencia do entorno familiar e do grupo social no tabagismo entre jovens brasileiros de 15 a 24 anos. Rev Panam Salud Publica 2011;30(1):22-30.

83. Malta DC, Sardinha LMV, Mendes I, Barreto SM, Giatti L, Castro IRR, et al. Prevalência de fatores de risco e proteção de doenças crônicas não transmissíveis em adolescentes: resultados da Pesquisa Nacional de Saúde do Escolar (PeNSE), Brasil, 2009. Cien Saude Colet 2010;15(suppl 2):3009-19. 
84. Hallal PC, Knuth AG, Cruz DKA, Mendes MI, Malta DC. Physical activity practice among Brazilian adolescents. Cien Saude Colet 2010;15(2):3035-42.

85. Andaki ACR, Tinôco ALA, Mendes EL, Júnior RA, Hills AP, Amorim PRS. Anthropometry and physical activity level in the prediction of metabolic syndrome in children. Public Health Nutr 2013;17(10):2287-94.

86. Neto FA, Eto FN, Pereira TSS, Carletti L, Molina MDCB. Active and sedentary behaviours in children aged 7 to 10 years old: The urban and rural contexts, Brazil. BMC Public Health 2014;14:1174.

87. Arruda ELM, Lopes AS. Gordura corporal, nível de atividade física e hábitos alimentares de adolescentes da região serrana de Santa Catarina, Brasil. Rev Bras Cineantropom Desempenho Hum 2007;9(1):05-11.

88. Ceschini FL, Andrade DR, Oliveira LC, Júnior JFA, Matsudo VKR. Prevalence of physical inactivity and associated factors among high school students from state's public schools. J Pediatr 2009;85(4):301-6.

89. Coelho LG, Cândido APC, Machado-Coelho GLL, Freitas SN. Association between nutritional status, food habits and physical activity level in schoolchildren. J Pediatr 2012;88(5):406-12.

90. Freire RS, Lélis FLO, Fonseca Filho JA, Nepomuceno MO, Silveira MF. Prática regular de atividade física: estudo de base populacional no Norte de Minas Gerais, Brasil. Rev Bras Med Esporte 2014;20(5):345-9.

91. Meireles AL, Xavier CC, Proietti FA, Caiaffa WT. Influence of individual and socio-environmental factors on self-rated health in adolescents. Rev Bras Epidemiol 2015;18(3):538-51.

92. Nakamura PM, Teixeira IP, Papini CB, Lemos N, Nazario MES, Kokubun E. Physical education in schools, sport activity and total physical activity in adolescents. Rev Bras Cineantropom Desempenho Hum 2013;15(5):517-27.

93. Peres SV, Latorre MRDO, Slater B, Tanaka LF, Silva MV. prevalência de excesso de peso e seus fatores associados em adolescentes da rede de ensino público de Piracicaba, São paulo. Rev Paul Pediatr 2012;30(1):57-64.

94. Romero A, Slater B, Florindo AA, Latorre MRDO, Cezar C, Silva MV. Determinantes do índice de massa corporal em adolescentes de escolas públicas de Piracicaba, São Paulo. Cien Saude Colet 2010;15(1):141-9.

95. Salvador CCZ, Kitoko PM, Gambardella AMD. Estado nutricional de crianças e adolescentes: fatores associados ao excesso de peso e acúmulo de gordura. Rev Bras Crescimento Desenvolv Hum 2014;24(3):313-9.

96. Santos A, Oliveira L, Farias Júnior J, Silva P, Silva E, Freitas C. Associação entre prática religiosa e comportamentos de risco à saúde em adolescentes de Pernambuco, Brasil. Rev Bras Ativ Fís Saúde 2015;20(3):284-96.

97. Silva DAS, Lima JDO, Silva RJS, Prado RL. Nível de atividade física e comportamento sedentário em escolares. Rev Bras Cineantropom Desempenho Hum 2009;11(3):299-306.

98. Souza RP. Nível de sedentarismo entre adolescentes do município de Canoas, Rio Grande do Sul, Brasil. Educ Fís Rev 2011;5(2):1-11.

99. Straatmann VS, Veiga GV. Cardiorespiratory fitness, physical activity, and indicators of adiposity in Brazilian adolescents. Hum Mov 2015;16(2):64-70.

100. Vieira CENK, Mariz LS, Dantas DNA, Menezes DJC, Rêgo MCD, Enders BC. Association between risk factors for hypertension and the Nursing Diagnosis overweight in adolescents. Investig Educ Enfermería 2016;34(2):305-13.

101. Vitta A, Barros DS, Palma R, Vinícius M, Soares F, Helena M, et al. Prevalência e fatores associados à adiposidade central e periférica em estudantes do ensino fundamental brasileiro. Rev Bras Crescimento Desenvolv Hum 2013;23(3):365-71.

102. Matsudo SM, Matsudo VR, Araújo T, Andrade D, Andrade E, Oliveira L. Nível de atividade física da população do Estado de São Paulo: análise de acordo com o gênero, idade, nível socioeconômico, distribuição geográfica e de conhecimento. Rev Bras Ciên Mov 2002;10(4):41-50. 
103. Nascimento-Ferreira M V., Collese TS, Moraes ACF, Rendo-Urteaga T, Moreno LA, Carvalho HB. Validity and reliability of sleep time questionnaires in children and adolescents: A systematic review and meta-analysis. Sleep Med Rev 2016;30(1):85-96.

104. Ramires V, Becker L, Sadovsky A, Zago A, Bielemann R, Guerra P. Evolução da pesquisa epidemiológica em atividade física e comportamento sedentário no Brasil: atualização de uma revisão sistemática. Rev Bras Ativ Fís Saúde 2014;19(5):529-47.

105. Dumith SC. Physical activity in Brazil: A systematic rewiew. Cad Saude Publica 2009;25(Suppl. 3):415-26.

106. Ndahimana D, Kim E-K. Measurement Methods for Physical Activity and Energy Expenditure: a Review. Clin Nutr Res 2017;6(2):68-80.

107. Moraes ACF, Guerra PH, Menezes PR. The worldwide prevalence of insufficient physical activity in adolescents; a systematic review. Nutr Hosp 2013;28(3):575-84.

108. Barufaldi LA, Abreu GDA, Coutinho ESF, Bloch KV. Meta-analysis of the prevalence of physical inactivity among Brazilian adolescents. Cad Saude Publica 2012;28(6):1019-32.

109. Barbosa Filho VC, Campos W, Lopes AS. Epidemiology of physical inactivity, sedentary behaviors, and unhealthy eating habits among Brazilian adolescents. Cien Saude Colet 2014;19(1):173-94.

\section{CORRESPONDING AUTHOR}

Valter Cordeiro Barbosa Filho Federal Institute of Education, Science and Techonology of Ceará, Boa Viagem

Campus. Rod. Pres. Juscelino Kubitschek, Boa Viagem - CE, 63870-000, Brazil

E-mail: valtercbf@gmail.com

\section{SUPPLEMENTARY FILE}

Example of descriptors used during the database search.

\begin{tabular}{|c|c|c|}
\hline Number & Groups & Descriptors \\
\hline$\# 1$ & $\begin{array}{l}\text { Physical activity } \\
\text { (outcome) }\end{array}$ & $\begin{array}{l}\text { ("physical activity" [Text Word] OR "motor activity" [MeSH } \\
\text { Terms] OR "physical exercise" [Text Word] OR exercise } \\
\text { [MeSH Terms] OR "exercise program" [Text Word] OR sports } \\
\text { [MeSH Terms] OR sport [Text Word] OR leisure activit* [Text } \\
\text { Word] OR recreation [Text Word]) }\end{array}$ \\
\hline \#2 & $\begin{array}{l}\text { Evaluation meth- } \\
\text { ods }\end{array}$ & $\begin{array}{l}\text { (self-report OR checklist OR recall OR } 24 \mathrm{~h} \text { OR interviews } \\
\text { OR questionnaire OR diary OR assessment OR survey OR } \\
\text { measurement OR Acceleromet }{ }^{\star} \text { OR pedomet OR inclinomet } \\
\text { OR (objectiv AND measur*) OR (direct* AND measure*)) }\end{array}$ \\
\hline \#3 & $\begin{array}{l}\text { Population (young } \\
\text { people) }\end{array}$ & $\begin{array}{l}\text { (youth [Text Word] OR teenage [Text Word] OR adolescent } \\
\text { [MeSH Terms] OR adolescent [Text Word] OR adolescence } \\
\text { [Text Word] OR students [MeSH Terms] OR student [Text } \\
\text { Word] OR child [Text Word] OR children [Text Word] OR } \\
\text { "young people" [Text Word]) }\end{array}$ \\
\hline \#4 & Context (country) & (Brazil* OR Brazilian) \\
\hline \multicolumn{2}{|c|}{ Final search } & \#1 AND \#2 AND \#3 AND \#4 \\
\hline
\end{tabular}

